\title{
Nonperturbative Dynamical Casimir Effect in Optomechanical Systems: Vacuum Casimir-Rabi Splittings
}

\author{
Vincenzo Macrì, ${ }^{1,2}$ Alessandro Ridolfo, ${ }^{2}$ Omar Di Stefano, ${ }^{2}$ Anton Frisk Kockum, ${ }^{2}$ Franco Nori,,${ }^{2,3}$ and Salvatore Savasta ${ }^{1,2}$ \\ ${ }^{1}$ Dipartimento di Scienze Matematiche e Informatiche, Scienze Fisiche e Scienze della Terra, \\ Università di Messina, I-98166 Messina, Italy \\ ${ }^{2}$ Center for Emergent Matter Science, RIKEN, Saitama 351-0198, Japan \\ ${ }^{3}$ Physics Department, The University of Michigan, Ann Arbor, Michigan 48109-1040, USA
}

(Received 10 July 2017; revised manuscript received 9 September 2017; published 26 February 2018)

\begin{abstract}
We study the dynamical Casimir effect using a fully quantum-mechanical description of both the cavity field and the oscillating mirror. We do not linearize the dynamics, nor do we adopt any parametric or perturbative approximation. By numerically diagonalizing the full optomechanical Hamiltonian, we show that the resonant generation of photons from the vacuum is determined by a ladder of mirror-field vacuum Rabi splittings. We find that vacuum emission can originate from the free evolution of an initial pure mechanical excited state, in analogy with the spontaneous emission from excited atoms. By considering a coherent drive of the mirror, using a master-equation approach to take losses into account, we are able to study the dynamical Casimir effect for optomechanical coupling strengths ranging from weak to ultrastrong. We find that a resonant production of photons out of the vacuum can be observed even for mechanical frequencies lower than the cavity-mode frequency. Since high mechanical frequencies, which are hard to achieve experimentally, were thought to be imperative for realizing the dynamical Casimir effect, this result removes one of the major obstacles for the observation of this long-sought effect. We also find that the dynamical Casimir effect can create entanglement between the oscillating mirror and the radiation produced by its motion in the vacuum field, and that vacuum Casimir-Rabi oscillations can occur. Finally, we also show that all these findings apply not only to optomechanical systems, but also to parametric amplifiers operating in the fully quantum regime.
\end{abstract}

DOI: 10.1103/PhysRevX.8.011031

\section{INTRODUCTION}

Quantum field theory predicts that vacuum fluctuations can be converted into real particles by the energy provided through certain external perturbations [1-8]. Examples include the Schwinger effect [1], predicting the production of electron-positron pairs from the vacuum under the application of intense electrical fields; Hawking radiation $[3,9]$, which is caused by the bending of space-time in intense gravitational fields and determines the evaporation of black holes; the Unruh effect [10], predicting that an accelerating observer will observe blackbody radiation where an inertial observer would observe none; and the dynamical Casimir effect (DCE) [2,6,7], describing the generation of photons from the quantum vacuum due to rapid changes of the geometry (in particular, the positions

Published by the American Physical Society under the terms of the Creative Commons Attribution 4.0 International license. Further distribution of this work must maintain attribution to the author(s) and the published article's title, journal citation, and DOI.
Subject Areas: Quantum Physics

of some boundaries) or material properties of electrically neutral macroscopic or mesoscopic objects.

The creation of photons by moving mirrors was first predicted by Moore [2] in 1970, for a one-dimensional cavity. In 1976, Fulling and Davies [11] demonstrated that photons can be generated even by a single mirror, when it is subjected to a nonuniform acceleration. Since the first prediction of the DCE, many different experimental setups, able to produce sudden nonadiabatic changes inducing light emission from the quantum vacuum, have been proposed [12]. These proposals can be divided into two main groups: setups where the photons are created due to the movement of mirrors [mechanical (M)DCE] [11,13-17], and systems where the boundary conditions are modulated by some effective motion producing a parametric amplification of vacuum fluctuations [parametric (P) DCE] [6-8,18-25].

The experimental detection of the DCE is challenging owing to the difficulty in changing the boundary conditions, e.g., by moving physical objects, such as massive mirrors, sufficiently fast for generation of a significant number of photons. In 1996, Lambrecht, Jaekel, and Reynaud [14] provided a quantitative estimate of the 


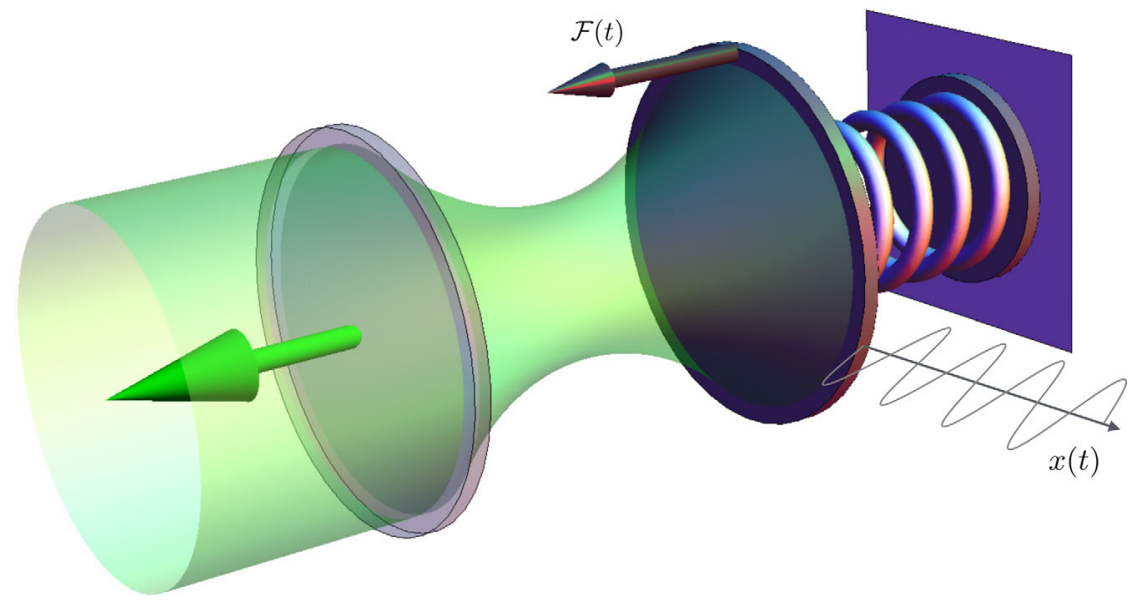

FIG. 1. Schematic of a generic optomechanical system where one of the mirrors in an optical cavity can vibrate at frequency $\omega_{m}$. If the vibrating mirror is excited by an external drive $\mathcal{F}(t)$, able to create a $k$-phonon state with a non-negligible probability, the vibrating mirror can emit photon pairs if $k \omega_{m} \simeq 2 \omega_{c}$, where $\omega_{c}$ is the resonance frequency of the cavity.

photon flux radiated from an optomechanical system consisting of a cavity with oscillating mirrors. Taking advantage of resonance-enhancement effects, they showed that a significant number of microwave photons, sufficient to allow detection, can be produced in realistic high- $Q$ cavities with a moderate peak velocity of the mirrors. However, the resonance condition, requiring that the mechanical oscillation frequency $\omega_{m}$ be at least twice that of the lowest-frequency cavity mode $\omega_{c}$, remains a major barrier to the experimental demonstration of the MDCE. Recently, high-frequency mechanical oscillators $\left[\omega_{m} /(2 \pi) \sim 6 \mathrm{GHz}\right]$ [26] have been realized. However, to produce vacuum radiation at a frequency $\omega_{c} /(2 \pi) \sim 5 \mathrm{GHz}$, a still higher mechanical frequency, $\omega_{m} /(2 \pi) \sim 10 \mathrm{GHz}$, is required.

In order to circumvent these difficulties, a number of theoretical proposals have suggested to use experimental setups where the boundary conditions are modulated by some effective motion instead (PDCE). Examples of such proposals include (i) using lasers to rapidly modulate the reflectivity of thin semiconductor films [18,20,21], (ii) modulating the resonance frequency of a superconducting stripline resonator [22], nonadiabatic time modulation of (iii) the light-matter coupling strength in cavity QED systems [23,27-33] or (iv) of the background in which the field propagates $[5,7,19]$, and (v) using a superconducting quantum interference device (SQUID) to modulate the boundary condition of a superconducting waveguide or resonator $[6,24,25]$.

Recently, using superconducting circuits [34-37], the DCE (specifically, the PDCE) has been demonstrated experimentally, implementing proposals (iv) and (v). In particular, it was observed [6] in a coplanar transmission line terminated by a SQUID whose inductance was modulated at high frequency $(>10 \mathrm{GHz})$. This was also demonstrated by modulating the index of refraction of a
Josephson metamaterial embedded in a microwave cavity [7]. Of course, these all-optical experiments do not demonstrate the conversion of mechanical energy into photons, as predicted by the DCE. Such a direct observation is still lacking.

Moreover, it is worth noting that the interpretation of these all-optical experiments is not unique. Although these experiments demonstrate key features of the DCE physics, the question may arise if these can be regarded as direct observations of the DCE or quantum simulations of the DCE. Specifically, these experiments can also be considered as examples of spontaneous parametric downconversion [38-40] in a waveguide or in a cavity (see, e.g., Ref. [37] for implementations with superconducting quantum circuits). In this optical quantum process, incident photons at frequency $\omega$ are converted into pairs of photons at lower frequencies $\omega_{1}$ and $\omega_{2}$, such that $\omega_{1}+\omega_{2}=\omega$. This process is determined by the nonlinearities of the material system and the vacuum fluctuations of the electromagnetic field. The validity of this description is confirmed by looking at the interaction Hamiltonians describing the investigated systems [7,24,25]. In both cases, they can be written as $\lambda\left(\delta \Phi_{\text {ext }} / \Phi_{0}\right) \Phi^{2}$, where $\Phi_{\text {ext }}$ is the external driving flux, $\Phi_{0}$ is the flux quantum, $\Phi$ is the flux field in the waveguide or in the cavity, and $\lambda$ is a coupling coefficient depending on the specific system. In this Hamiltonian, the amplitude of the driving field is multiplied with the square of the amplitude of the excited field, just like in the Hamiltonians that describe secondorder nonlinear optical processes. Furthermore, conceptually there are subtle differences between moving boundaries and moving sources (see, e.g., Ref. [41]). We also observe that other proposed all-optical PDCE setups [such as (i) and (iii)] might have undesired background signals, due to the undesired excitations arising from the driving fields which can overwhelm the expected signal (see, e.g., 
Ref. [42]). Hence, direct observations of the conversion of mechanical energy into photon pairs (MDCE) would be highly desirable.

Most theoretical studies of the MDCE consider the mirror that scatters the vacuum field to follow a prescribed motion [2,11-13,15,43-45]. Therefore, the photon creation from the initial vacuum state is usually described as a parametric amplification process, just as in the case of the PDCE. Exceptions consider fluctuations of the position of the mirror driven by vacuum radiation pressure using linear dispersion theory $[46,47]$ or focus on the mirror motion as the main dynamical degree of freedom (d.o.f.). In the latter case, studies have shown how the DCE induces friction forces on the mirror $[48,49]$ or leads to decoherence of mechanical quantum superposition states [50].

In this article, we investigate the MDCE in cavityoptomechanical systems [51], treating both the cavity field and the moving mirror as quantum-mechanical systems. Calculations are made without performing any linearization of the dynamical equations. Multiple scattering between the two subsystems is taken into account nonperturbatively. The interactions of the two subsystems with the environment is described by using a master equation [52,53]. A surprising feature of this approach is that the DCE can be described without the need for a time-dependent lightmatter interaction. The only time-dependent Hamiltonian term considered here is the one describing the external drive of the vibrating mirror. Actually, within this approach, the DCE effect can be described, at least in principle, even without considering any time-dependent Hamiltonian. Specifically, we find that vacuum radiation can originate from the free evolution of an initial pure mechanical excited state, in analogy with the spontaneous emission from excited atoms. We believe that this theoretical framework provides a more fundamental explanation of the DCE. Note that fundamental processes in quantum field theory are described by interaction Hamiltonians which do not depend parametrically on time.

We find that the resonant generation of photons from the vacuum is determined by a ladder of mirror-field vacuum Rabi-like energy splittings. When the loss rates are lower than the corresponding frequency splittings, a reversible exchange of energy between the vibrating mirror and the cavity field, which we call vacuum Casimir-Rabi oscillations, can be observed.

Cavity-optomechanics experiments are rapidly approaching the regime where the radiation pressure of a single photon displaces the mechanical oscillator by more than its zero-point uncertainty [54-60]. Specifically, in circuit optomechanics, it has been shown that the radiation pressure effect can be strongly enhanced by introducing a qubit-mediated [57,58] or modulated [61] interaction between the mechanical and the electromagnetic resonator. This ultrastrong-coupling (USC) regime, where the optomechanical coupling rate is comparable to the mechanical frequency, can give rise to strong nonlinearities even in systems described by the standard optomechanics interaction Hamiltonian, which depends linearly on the mirror displacement $[62,63]$. This regime favors the observation of macroscopic mechanical oscillators in a nonclassical state of motion [62-65]. This requires a full quantum treatment of both the mechanical and optical d.o.f., and multiplescattering effects between the field and the mechanical oscillator cannot be ignored.

The approach considered here allows us to extend the investigation of the DCE to the USC limit of cavity optomechanics. We find that this regime is able to remove one of the major obstacles for the experimental observation of this long-sought effect. Indeed, we show that, approaching USC, a resonant production of photons out from the vacuum can be observed for mechanical frequencies lower than the lowest cavity-mode frequency. Approximately, the resonance condition for the production of photon pairs out from the vacuum is $k \omega_{m} \simeq 2 \omega_{c}$, with $k$ integer. This corresponds to processes where $k$ phonons in the mechanical oscillator are converted into two cavity photons. The matrix element for this transition decreases rapidly for increasing $k$, but increases when the optomechanical coupling $g$ increases. Already the resonance condition with $k=2$, corresponding to $\omega_{m} \simeq \omega_{c}$, where DCE matrix elements display reasonable amplitude even at moderate coupling, is promising for the observation of the MDCE. Indeed, this resonance condition can be achieved in the $\mathrm{GHz}$ spectral range using ultrahigh-frequency mechanical micro- or nanoresonators [26,60].

Very recently, new resonance conditions in the DCE that potentially allow the production of photons for $\omega_{m}<\omega_{c}$ have been found assuming a classical prescribed anharmonic motion of the mirror [66]. This model, however, describes photon emission from vacuum fluctuations only in the instability region, and the resulting time evolution of the mean photon number grows exponentially even in the presence of cavity losses. On the contrary, in our approach, the vibrating mirror is treated as a harmonic (anharmonicity originates only from the interaction) quantum d.o.f. on the same footing as the cavity field and we do not find unstable regions.

\section{RESULTS}

\section{A. Model}

We consider the case of a cavity with a movable end mirror (see Fig. 1) and focus on the simplest possible model system in cavity optomechanics, which has been used to successfully describe most of such experiments to date. A detailed derivation of the optomechanical Hamiltonian can be found in Ref. [67]. Both the cavity field and the position of the mirror are treated as dynamical variables and a canonical quantization procedure is adopted. By considering only one mechanical mode with resonance frequency 
$\omega_{m}$ and bosonic operators $\hat{b}$ and $\hat{b}^{\dagger}$, and only the lowestfrequency optical mode $\omega_{c}$ of the cavity, with bosonic operators $\hat{a}$ and $\hat{a}^{\dagger}$, the system Hamiltonian can be written as $\hat{H}_{s}=\hat{H}_{0}+\hat{H}_{I}$, where

$$
\hat{H}_{0}=\hbar \omega_{c} \hat{a}^{\dagger} \hat{a}+\hbar \omega_{m} \hat{b}^{\dagger} \hat{b}
$$

is the unperturbed Hamiltonian. The Hamiltonian describing the mirror-field interaction is

$$
\hat{H}_{I}=\frac{\hbar G}{2}\left(\hat{a}+\hat{a}^{\dagger}\right)^{2} \hat{x},
$$

where $\hat{x}=x_{\mathrm{ZPF}}\left(\hat{b}+\hat{b}^{\dagger}\right)$ is the mechanical displacement ( $x_{\mathrm{ZPF}}$ is the zero-point-fluctuation amplitude of the vibrating mirror) and $G$ is a coupling parameter. By developing the photonic factor in normal order, and by defining new bosonic phonon and photon operators and a renormalized photon frequency, $\hat{H}_{s}$ can be written as

$$
\hat{H}_{s}=\hat{H}_{0}+\hat{V}_{\mathrm{om}}+\hat{V}_{\mathrm{DCE}}
$$

where $\hat{H}_{0}$ formally coincides with Eq. (1),

$$
\hat{V}_{\mathrm{om}}=\hbar g \hat{a}^{\dagger} \hat{a}\left(\hat{b}+\hat{b}^{\dagger}\right)
$$

is the standard optomechanical interaction conserving the number of photons, and

$$
\hat{V}_{\mathrm{DCE}}=\frac{\hbar g}{2}\left(\hat{a}^{2}+\hat{a}^{\dagger 2}\right)\left(\hat{b}+\hat{b}^{\dagger}\right)
$$

describes the creation and annihilation of photon pairs [68], where $g=G x_{\mathrm{ZPF}}$ is the optomechanical coupling rate. As we see in detail below, $\hat{V}_{\text {DCE }}$ determines the DCE. The Hamiltonian Eq. (3) describes the interaction between a moving mirror and the radiation pressure of a cavity field [69]. However, the same radiation-pressure-type coupling is obtained for microwave optomechanical circuits (see, e.g., Refs. [57,59]). In the following, we use the Hamiltonian in Eq. (3) including only one optical mode. In many circuit-optomechanics experiments, the electromagnetic resonator is provided by a superconducting $L C$ circuit, which supports only a single mode.

The results we present here can be generalized to include additional modes, even if the computational effort will increase (see Appendix D). We expect that higher-energy additional modes, when present, can be excited at driving amplitudes higher than those considered here.

When describing most of the optomechanics experiments to date [51], $\hat{V}_{\mathrm{DCE}}$ is neglected. This is a very good approximation when the mechanical frequency is much smaller than the cavity frequency (which is the most common experimental situation), since $\hat{V}_{\text {DCE }}$ connects bare states with an energy difference $2 \hbar \omega_{c} \pm \hbar \omega_{m}$ much larger than the coupling strength $\hbar g$. With this approximation, the resulting Hamiltonian, $\hat{H}_{0}+\hat{V}_{\text {om }}$, conserves the number of photons and can be analytically diagonalized. The full Hamiltonian in Eq. (3) provides the simplest unified description of cavity-optomechanics experiments and the DCE in a cavity with a vibrating mirror.

In order to properly describe the system dynamics, including external driving, dissipation and decoherence, the coupling to external d.o.f. needs to be considered. A coherent external drive of the vibrating mirror can be described by including the following time-dependent Hamiltonian:

$$
\hat{V}_{m}(t)=\mathcal{F}(t)\left(\hat{b}+\hat{b}^{\dagger}\right),
$$

where $\mathcal{F}(t)$ is proportional to the external force applied to the mirror. Analogously, the coherent optical excitation of the cavity mode can be described by

$$
\hat{V}_{c}(t)=\mathcal{E}(t)\left(\hat{a}+\hat{a}^{\dagger}\right)
$$

where $\mathcal{E}(t)$ is proportional to the coherent optical field exciting the cavity. In the following, we only consider the external excitation of the mirror $[\mathcal{E}(t)=0]$ by a continuous-wave drive or by a pulse, in contrast to most cavityoptomechanical experiments, where the system is optically excited.

\section{B. Vacuum Casimir-Rabi splittings}

We begin by numerically diagonalizing the system Hamiltonian $\hat{H}_{s}$ in Eq. (3). Figure 2(a) displays the lowest energy levels as a function of the ratio between the cavity and the mechanical frequency, using an optomechanical coupling $g / \omega_{m}=0.04$. For comparison, we also show in Fig. 2(a) (dashed gray lines) the lowest energy levels,

$$
E_{n, k}=\hbar \omega_{c} n-\hbar g^{2} n^{2} / \omega_{m}+\hbar \omega_{m} k
$$

for the standard optomechanics Hamiltonian $\hat{H}_{0}+\hat{V}_{\text {om }}$. In this case, the system eigenstates can be written as

$$
\left|n, k_{n}\right\rangle=|n\rangle_{c} \otimes \hat{D}(n \beta)|k\rangle_{m}
$$

where $n$ is the cavity photon number and the mechanical state $\left|k_{n}\right\rangle$ is a displaced Fock state, determined by the displacement operator $\hat{D}(n \beta)=\exp \left[n \beta\left(\hat{b}^{\dagger}-\hat{b}\right)\right]$, with $\beta=$ $g / \omega_{m}$. The dashed gray horizontal lines in Fig. 2(a) correspond to states $\left|0, k_{0}\right\rangle \equiv|0, k\rangle$ belonging to the $n=0$ manifold. The dashed gray lines with lower nonzero slope (slope 1 ) describe the $n=1$ manifold $\left(\left|1, k_{1}\right\rangle\right)$, while those with slope 2 describe the energy levels of the manifold with $n=2$. 

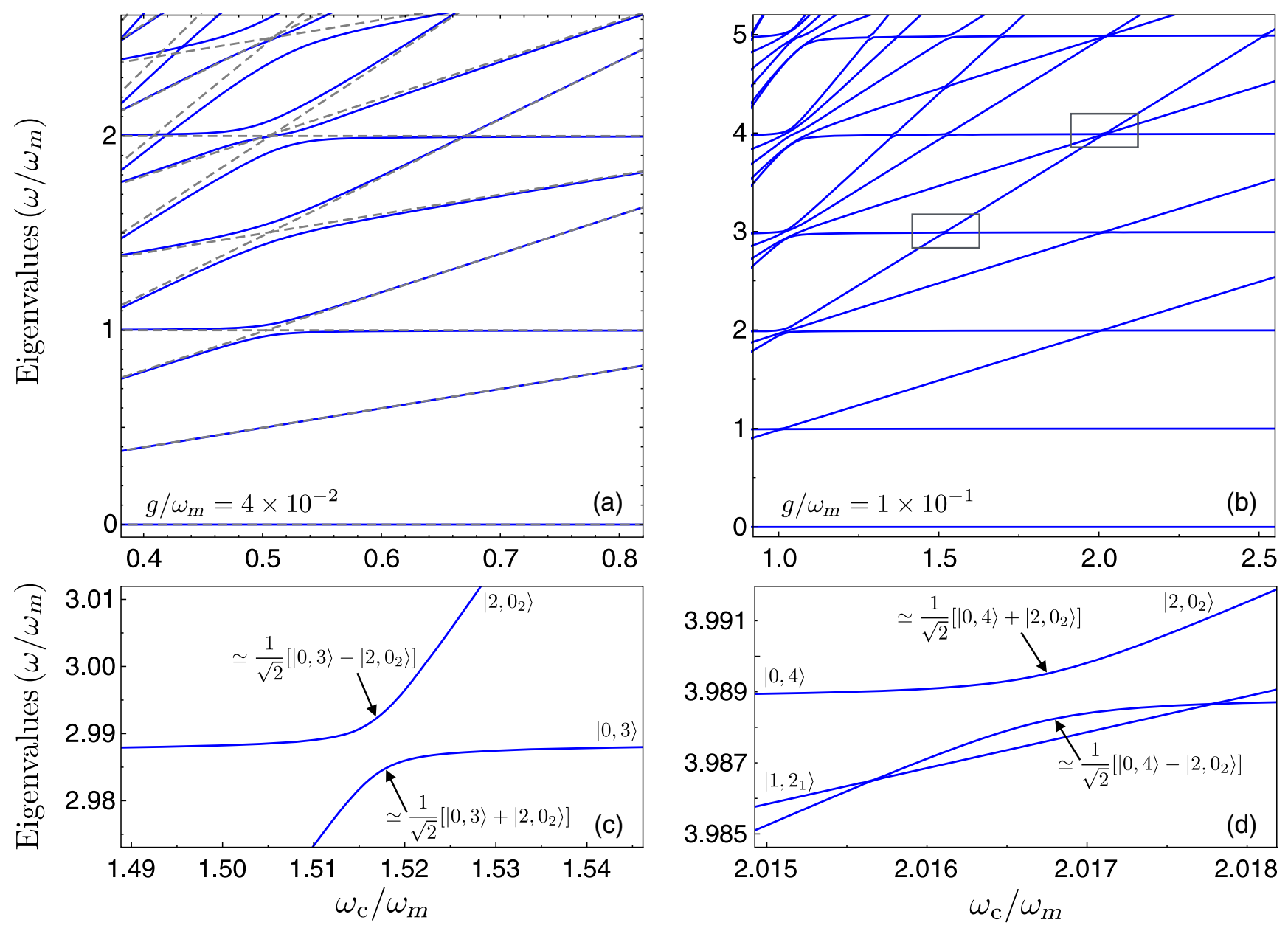

FIG. 2. Lowest-energy levels of the system Hamiltonian as a function of the ratio between the cavity frequency and the mechanical frequency. In (a) an optomechanical coupling $g / \omega_{m}=0.04$ has been used. The dashed gray lines describe the eigenenergies of the standard optomechanics Hamiltonian $\hat{H}_{0}+\hat{V}_{\text {om }}$. The blue continuous curves are the eigenvalues of $\hat{H}_{s}=H_{0}+\hat{V}_{\text {om }}+\hat{V}_{\mathrm{DCE}}$, which have also been calculated for a coupling $g / \omega_{m}=0.1$, as shown in (b). Panels (c) and (d) display enlarged views of the two boxed regions in (b) showing avoided-level crossings due to optomechanical hybridizations of zero- and two-photon states (vacuum Casimir-Rabi splittings).

The continuous blue lines correspond to the energy levels obtained by numerically diagonalizing the system Hamiltonian $\hat{H}_{s}$ in Eq. (3). The main difference compared to the gray lines is the appearance of level anticrossings of increasing size at increasing eigenenergy values when $E_{0, k}=E_{2, k-1}$ (corresponding to a cavity frequency $\left.\omega_{c}=\omega_{m} / 2+2 g^{2} / \omega_{m} \simeq \omega_{m} / 2\right)$. We observe that the condition $\omega_{c} \simeq \omega_{m} / 2$ is the standard resonance condition $\left(\omega_{m}=2 N \omega_{c}\right)$ for the DCE in a cavity with a vibrating mirror [14], with $N=1$. These avoided crossings arise from the coherent coupling induced by $\hat{V}_{\text {DCE }}$ between the states $|0, k\rangle \leftrightarrow\left|2,(k-1)_{2}\right\rangle$ with $k \geq 1$. If the optomechanical coupling is not too strong, the size of the anticrossings can be analytically calculated by using first-order perturbation theory. By approximating $\left|k_{2}\right\rangle \simeq|k\rangle$, for the energy splittings, we obtain the simple expression

$$
2 \hbar \Omega_{0, k}^{2, k-1}=2\left\langle 2,(k-1)_{2}\left|\hat{V}_{\mathrm{DCE}}\right| 0, k\right\rangle \simeq \hbar g \sqrt{2 k},
$$

in very good agreement with the numerical results in Fig. 2(a). When the splitting is at its minimum $\left(\omega_{c}=\omega_{m} / 2+2 g^{2} / \omega_{m}\right)$, the two system eigenstates are approximately (not exactly, owing to dressing effects induced by $\hat{V}_{\text {DCE }}$ ) the symmetric and antisymmetric superposition states

$$
\left|\psi_{2(3)}\right\rangle \simeq \frac{1}{\sqrt{2}}\left(|0,1\rangle \pm\left|2,0_{2}\right\rangle\right) .
$$

These vacuum Casimir-Rabi splittings, demonstrating optomechanical-induced hybridization of zero- and twophoton states, establish a close analogy between the DCE and cavity QED, where the atom-photon vacuum Rabi splitting and quantum Rabi oscillations in the time domain have been observed in many systems and widely exploited for many applications [70]. We observe, however, that, while quantum Rabi splittings in cavity QED describe coherent coupling between states with the same number of 
excitations, Casimir-Rabi splittings involve pairs of states with different number of excitations. In this case, for example, a state with $k$ excitations (phonons) hybridizes with a state with $k+1$ excitations ( $k-1$ phonons and 2 photons). This nonconservation of excitation numbers is reminiscent of cavity QED in the USC regime, where the counterrotating terms in the atom-field interaction Hamiltonian enable the coupling of states with different excitation numbers [71-76]. More generally, the DCE Hamiltonian in Eq. (5) gives rise to several additional avoided-level crossings, describing resonant optomechanical scattering processes $\left|n, k_{n}\right\rangle \leftrightarrow\left|n+2,(k-q)_{n+2}\right\rangle$, which occur when the energies of the final and initial states coincide $\left(2 \omega_{c} \sim q \omega_{m}\right)$.

These coherent couplings induced by $\hat{V}_{\text {DCE }}$ constitute the fundamental quantum mechanism through which mechanical energy is transferred to the vacuum electromagnetic field, giving rise to the DCE. For example, in the absence of losses, an initial 1-phonon-0-photon state $|0,1\rangle$ (not being a system eigenstate) will evolve as

$$
|\psi(t)\rangle=\cos \left(\Omega_{0,1}^{2,0} t\right)|0,1\rangle-i \sin \left(\Omega_{0,1}^{2,0} t\right)\left|2,0_{2}\right\rangle,
$$

and thus, after a time $t=\pi /\left(2 \Omega_{0,1}^{2,0}\right)$, will spontaneously evolve into a photon pair. This elementary analysis shows that, if the mechanical and photonic loss rates are much lower than the coupling rate $\Omega_{0,1}^{2,0}$, mechanical energy can be converted, at least in principle, into light with $100 \%$ efficiency. Moreover, according to Eq. (10), at $t=\pi /\left(4 \Omega_{0,1}^{2,0}\right)$, the moving mirror and the cavity field become maximally entangled.

Figure 2(b) shows the lowest-energy eigenvalues of $\hat{H}_{s}$ for larger cavity-mode frequencies, using a stronger optomechanical coupling $g / \omega_{m}=0.1$. The figure [see also the boxed details enlarged in Figs. 2(c) and 2(d)] shows that optomechanical resonant couplings occur also for $\omega_{m}<2 \omega_{c}$. In particular, vacuum Casimir-Rabi splittings occur when $E_{0, k}=E_{2, k-q}$, corresponding to a cavity frequency $\omega_{c}-2 g^{2} / \omega_{m} \simeq q \omega_{m} / 2$, also when $q>1$. Avoided-level crossings for $q=2$ are clearly visible in Fig. 2(b). Smaller splittings for $q=3$ and $q=4$ are indicated by black boxes, and their enlarged views are shown in Figs. 2(c) and 2(d). By using first-order perturbation theory, the size of these avoided-level crossings can be calculated analytically:

$$
\begin{aligned}
2 \hbar \Omega_{0, k}^{2, k-q} & =2\left\langle 0, k\left|\hat{V}_{\mathrm{DCE}}\right| 2,(k-q)_{2}\right\rangle \\
& =\sqrt{2} \hbar g\left[\sqrt{k+1} D_{k+1, k-q}(2 \beta)+\sqrt{k} D_{k-1, k-q}(2 \beta)\right],
\end{aligned}
$$

where the matrix elements of the displacement operators can be expressed in terms of associated Laguerre polynomials: $\quad D_{k^{\prime}, k}(\alpha)=\sqrt{k ! / k^{\prime} !} \alpha^{k^{\prime}-k} e^{-|\alpha|^{2} / 2} L_{k}^{k^{\prime}-k}\left(|\alpha|^{2}\right)$. We note that the resonance conditions with $\omega_{m} \leq \omega_{c}$ have nonzero DCE matrix elements Eq. (11) thanks to the nonorthogonality of mechanical Fock states with different phonon numbers, belonging to different photonic manifolds:

$$
\left\langle k \mid k_{2}^{\prime}\right\rangle=D_{k, k^{\prime}}(2 \beta) \neq 0
$$

(see, e.g., Ref. [65]). Note also that, for $\beta \rightarrow 0$, $\left\langle k \mid k_{2}^{\prime}\right\rangle \rightarrow \delta_{k, k^{\prime}}$. Examples of analytically calculated splittings $2 \Omega_{0, k}^{2, k-q}$ are displayed in Appendix A, where we also present a comparison between the numerically calculated vacuum Rabi splitting and the corresponding analytical calculations, obtained with first-order perturbation theory for $2 \Omega_{0,3}^{2,0}$ and $2 \Omega_{0,4}^{2,0}$.

Also for resonance conditions with $q>1$, when the splitting is at its minimum (corresponding to values of $\omega_{c}$ such that $E_{0, k} \simeq E_{2, k-q}$ ), the two system eigenstates are essentially symmetric and antisymmetric linear superpositions. For example, for the boxed splitting at lower $\omega_{c}$,

$$
\left|\psi_{5(6)}\right\rangle \simeq \frac{1}{\sqrt{2}}\left(|0,3\rangle \pm\left|2,0_{2}\right\rangle\right) .
$$

Neglecting losses, an initial 3-phonon state $|0,3\rangle$ (not being a system eigenstate) will thus evolve spontaneously as

$$
|\psi(t)\rangle=\cos \left(\Omega_{0,3}^{2,0} t\right)|0,3\rangle-i \sin \left(\Omega_{0,3}^{2,0} t\right)|2,0\rangle,
$$

giving rise to a $100 \%$ mechanical-to-optical energy transfer and to vacuum-induced entanglement.

\section{DCE in the weak-coupling regime}

Here we investigate the dynamics giving rise to the DCE, numerically solving the system master equation (described in Appendix B). We focus on some experimentally promising cases, with $\omega_{c} \geq \omega_{m}$. In this section, we limit our investigations to the weak-coupling regime, which, however, does not refer to the optomechanical coupling strength (we use $g / \omega_{m}$ up to 0.1). Instead, following the terminology of cavity QED, by the term weak, we mean Casimir-Rabi splittings $2 \Omega_{0, k}^{2, k-q}$ smaller than the total decoherence rate $\gamma+\kappa$, where $\gamma$ and $\kappa$ are the mechanical and photonic loss rates, respectively (see Appendix B). We consider the optomechanical system initially in its ground state and numerically solve the master equation [Eq. (B1)] including the excitation of the moving mirror by a single-tone continuous-wave mechanical drive $\mathcal{F}(t)=A \cos \left(\omega_{d} t\right)$. Figure 3 shows the time evolution of the mean phonon number $\left\langle\hat{B}^{\dagger} \hat{B}\right\rangle$ (blue dash-dotted curve), the intracavity mean photon number $\left\langle\hat{A}^{\dagger} \hat{A}\right\rangle$ (black solid curve), and the equal-time photonic normalized second-order correlation function (red dashed curve), 


$$
g^{(2)}(t, t)=\frac{\left\langle\hat{A}^{\dagger}(t) \hat{A}^{\dagger}(t) \hat{A}(t) \hat{A}(t)\right\rangle}{\left\langle\hat{A}^{\dagger}(t) \hat{A}(t)\right\rangle^{2}},
$$

where $\hat{A}, \hat{B}$ are dressed photonic and phononic operators, as explained in Appendix B. We assume a zero-temperature reservoir and use $\kappa / \omega_{m}=3 \times 10^{-3}$ and $\gamma=10 \kappa$ for the photonic and mechanical loss rates. We consider a weak $(A / \gamma=2)$ resonant excitation of the vibrating mirror $\left(\omega_{d}=\omega_{m}\right)$. Figure 3 shows the system dynamics for the case $\omega_{c} \simeq 3 \omega_{m} / 2$ [corresponding to the minimum level splitting shown in Fig. 2(c)]. We use a normalized coupling $g / \omega_{m}=0.1$. The results demonstrate that a measurable rate of photons is produced. In particular, a steady-state mean intracavity photon number $\left\langle\hat{A}^{\dagger} \hat{A}\right\rangle_{\mathrm{ss}} \simeq 0.3$ is obtained, corresponding [for a resonance frequency of the cavity mode $\left.\omega_{c} /(2 \pi)=6 \mathrm{GHz}\right]$ to a steady-state output photon flux $\Phi=\kappa\left\langle\hat{A}^{\dagger} \hat{A}\right\rangle_{\mathrm{ss}} \sim 3 \times 10^{6}$ photons per second. The output photon flux is remarkable, taking into account the weak mechanical drive, corresponding to a steady-state mean phonon number $\left\langle\hat{b}^{\dagger} \hat{b}\right\rangle_{\mathrm{ss}}=4$ for $g / \omega_{m}=0$, and the quite low cavity quality factor $Q_{c}=\omega_{c} / \kappa=500$ used in the numerical calculations. Note that $Q_{c}$ values beyond $10^{6}$ are obtained in microwave resonators (see, e.g., Ref. [77]). Also, the mechanical loss rate $\gamma$ used here corresponds to a quality factor $Q_{m}$ one order of magnitude lower than the experimentally measured values in ultrahigh-frequency mechanical resonators $[26,60]$. Such a low driving amplitude and quality factors were used in order to reduce both memory and numerical effort. We observe that the steadystate phonon number does not reach the value $\left\langle\hat{B}^{\dagger} \hat{B}\right\rangle_{\mathrm{ss}}=4$ obtained in the absence of $\hat{V}_{\text {DCE }}$. This is an expected result, since the calculations fully take into account the correlated field-mirror dynamics induced by the DCE. The calculated

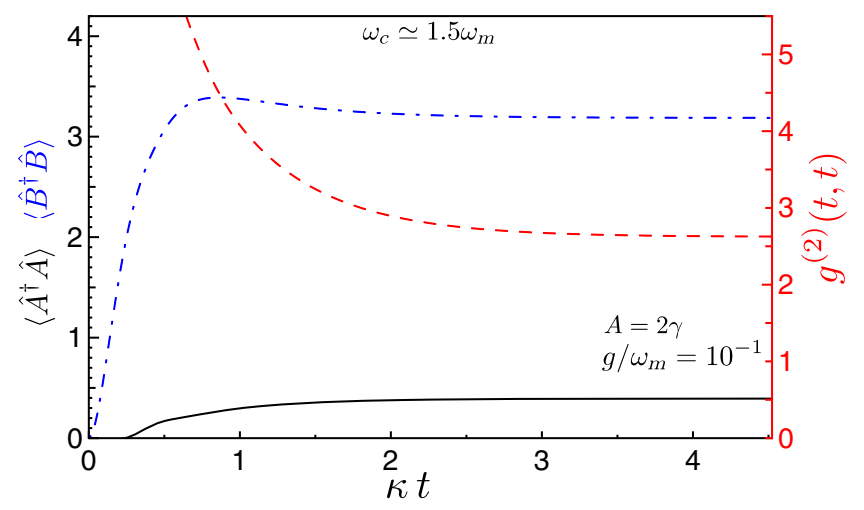

FIG. 3. System dynamics for $\omega_{c} \simeq 1.5 \omega_{m}$ under continuouswave drive of the vibrating mirror. The blue dash-dotted curve describes the mean phonon number $\left\langle\hat{B}^{\dagger} \hat{B}\right\rangle$, while the black solid curve describes the mean intracavity photon number $\left\langle\hat{A}^{\dagger} \hat{A}\right\rangle$ rising thanks to the DCE. The zero-delay normalized photon-photon correlation function $g^{(2)}(t, t)$ is also plotted as a red dashed curve with values given on the $y$ axis on the right. All parameters are given in the text. second-order correlation function $g^{(2)}(t, t)$, also displayed in Fig. 3, starts with very high values, confirming that photons are emitted in pairs. As time goes on, it decreases significantly, due to losses which affect the photon-photon correlation and to the increase in the mean photon number [note that $g^{(2)}(t, t)$, owing to the squared denominator, is intensity dependent].

Figure 4 displays results for the case $\omega_{c} \simeq \omega_{m}$. In this case, a higher-frequency mechanical oscillator is required. However, as we point out in the Introduction, mechanical oscillators with resonance frequencies $\omega_{m} /(2 \pi) \sim 6 \mathrm{GHz}$ have been realized [26]. In the present case, the DCE can be observed by coupling such a mechanical oscillator to a microwave resonator with the same resonance frequency. The advantage of this configuration is that the corresponding matrix elements (vacuum Casimir-Rabi splittings) $\Omega_{0, k}^{2, k-2}$ are non-negligible even for quite low optomechanical couplings. Figure 4(a), obtained using a coupling $g / \omega_{m}=0.1$, shows a remarkable energy transfer from the moving mirror to the cavity field which in its steady state contains more than 1 photon, corresponding to a

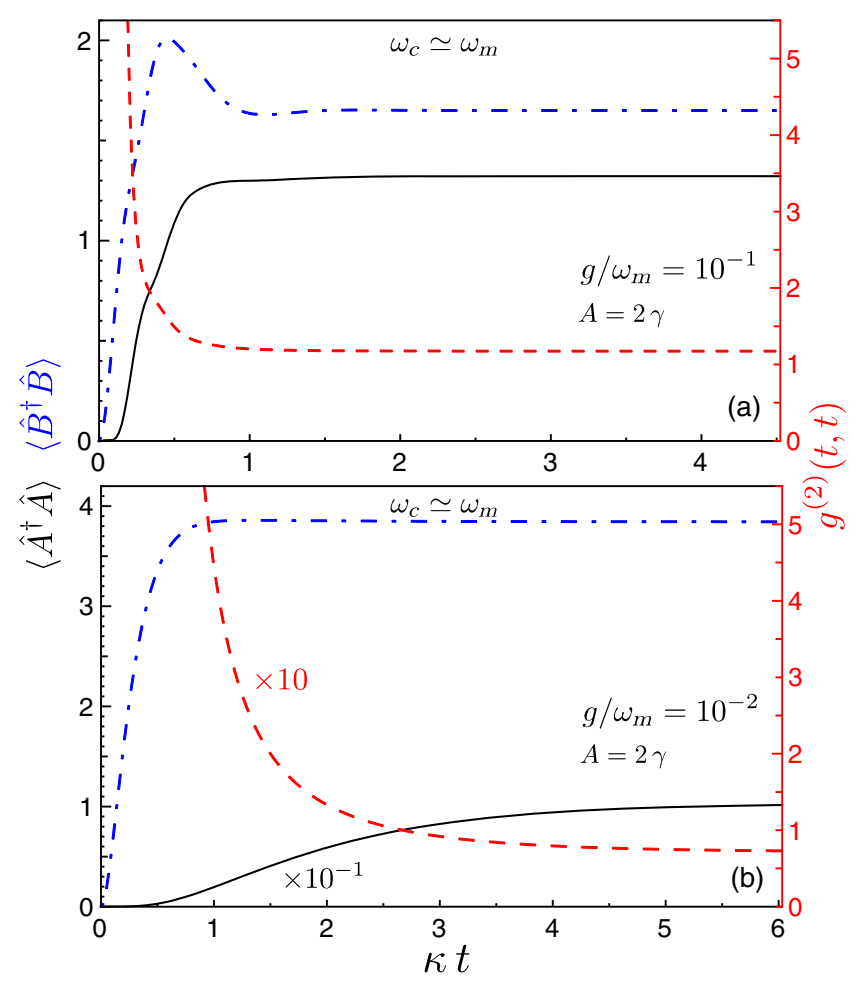

FIG. 4. System dynamics for $\omega_{c} \simeq \omega_{m}$ under continuous-wave drive of the vibrating mirror. The blue dash-dotted curves describe the mean phonon number $\left\langle\hat{B}^{\dagger} \hat{B}\right\rangle$, while the black solid curves describe the mean intracavity photon number $\left\langle\hat{A}^{\dagger} \hat{A}\right\rangle$ rising thanks to the DCE. The zero-delay normalized photon-photon correlation function $g^{(2)}(t, t)$ is also plotted (red dashed curve with values given on the $y$ axis on the right). Panel (a) has been obtained using $g / \omega_{m}=0.1$, panel (b) with $g / \omega_{m}=10^{-2}$. All the other parameters are given in the text. 
steady-state output photon flux $\Phi$ beyond $10^{7}$ photons per second. Figure 4(b) was obtained using an optomechanical coupling 1 order of magnitude lower. The resulting steadystate mean intracavity photon number decreases by 1 order of magnitude to $\left\langle\hat{A}^{\dagger} \hat{A}\right\rangle_{\text {ss }} \simeq 0.1$, but it is still measurable. This result is particularly interesting because it shows that the MDCE can be observed with state-of-the-art ultrahighfrequency mechanical resonators $[26,60]$ and with normalized coupling rates $\beta$ below those already achieved in circuit optomechanics [57], although with a lowerfrequency mechanical oscillator. We can conclude that the MDCE can be observed at $\omega_{c} \simeq \omega_{m}$ even when the optomechanical USC regime is not reached, although reaching it can significantly enhance the emission rate.

Quantum correlations in microwave radiation produced by the DCE in a superconducting waveguide terminated and modulated by a SQUID have been investigated [78]. The results indicate that the produced radiation can be strictly nonclassical and can display a measurable amount of intermode entanglement. In the approaches where the real or effective mirror is assumed to follow a prescribed classical motion, the entanglement between the moving mirror and the emitted electromagnetic field cannot be investigated. On the contrary, the present theoretical framework, fully taking into account the quantum correlations between the moving mirror and the cavity field, induced by $\hat{V}_{\mathrm{DCE}}$, allows us to investigate if the DCE creates optomechanical entanglement. In the present case, the dynamics involve many system states and, owing to the presence of losses, the system is far from being in a pure state during its time evolution. We quantify the entanglement using the negativity $\mathcal{N}$ (see Appendix C). By considering the same, numerically calculated, density matrix used to derive the results shown in Fig. 3, we find a steady-state negativity oscillating around $\mathcal{N} \simeq 5 \times 10^{-2}$, attesting that the DCE is able to produce mirror-field steady-state entanglement. For comparison, a maximally entangled Bell-like state, like that described by Eq. (13) at time $t=\pi /\left(4 \Omega_{0,3}^{2,0}\right)$, has a negativity $\mathcal{N}=0.5$. Using the parameters of Fig. 4(a), we find a larger steady-state negativity, oscillating around $\mathcal{N} \simeq 0.1$. However, we find no entanglement $(\mathcal{N} \simeq 0$ ) for the parameters of Fig. 4(b), when the influence of the DCE on the dynamics of the moving mirror is small.

\section{Vacuum Casimir-Rabi oscillations}

Here we investigate the DCE in the strong-coupling regime, when the Casimir-Rabi splittings $2 \Omega_{0, k}^{2, k-q}$ are larger than the total decoherence rate $\gamma+\kappa$. This regime is particularly interesting, since it provides direct evidence of the level structure determining the DCE and the multiplescattering effects between the two subsystems. Moreover, as we show, it gives rise to nonperturbative entangled dynamics of the cavity field and the moving mirror.
We numerically solve the master equation [Eq. (B1)], assuming the optomechanical system prepared in its ground state and including the vibrating mirror excitation by an ultrafast resonant pulse $\mathcal{F}(t)=\mathcal{A G}\left(t-t_{0}\right) \cos \left(\omega_{d} t\right)$, where $\mathcal{G}(t)$ is a normalized Gaussian function. We consider an optomechanical coupling $g / \omega_{m}=0.1$ and set the cavity frequency at the value providing the minimum level splitting $2 \Omega_{0,3}^{2,0} / \omega_{m} \simeq 8 \times 10^{-3}$ shown in Fig. 2(c) $\left(\omega_{c} \simeq 3 \omega_{m} / 2\right)$. We consider pulses with central frequency resonant with the mechanical oscillator $\left(\omega_{d}=\omega_{m}\right)$, and with standard deviation $\sigma=\left(20 \Omega_{0,3}^{2,0}\right)^{-1} \simeq 12 / \omega_{m}$. For the loss rates, we use $\gamma=0.15 \Omega_{0,3}^{2,0} \simeq 6 \times 10^{-4} \omega_{m}$ and $\kappa=\gamma / 2$.

Figure 5 displays the system dynamics after the pulse arrival and the Fourier transform of the mean photon number, obtained for pulses with amplitudes increasing from top to bottom: $\mathcal{A}=\pi / 3,2 \pi / 3, \pi$. Figures $5(\alpha)-5(\gamma)$ show (Casimir-Rabi) nutations (superimposed on the exponential decay due to the presence of losses) of the signals $\left\langle\hat{A}^{\dagger} \hat{A}\right\rangle$ (black solid curves) and $\left\langle\hat{B}^{\dagger} \hat{B}\right\rangle$ (blue dashed curves). The mean phonon number displays much less pronounced oscillations which are anticorrelated with the photonic oscillations. When the pulse amplitude is small $(A=\pi / 3)$, the time evolution of the mean photon number is sinusoidal-like with peak amplitudes decaying exponentially. Initially, the ultrafast kick produces a coherent mechanical state. The lowest Fock state in this mechanical coherent superposition, which is able to resonantly produce photon pairs, is $|0,3\rangle$ (see Fig. 2). This state is coherently coupled to the state $\left|2,0_{2}\right\rangle$ by $\hat{V}_{\text {DCE }}$, giving rise to the avoided-crossing states $\left|\psi_{5}\right\rangle$ and $\left|\psi_{6}\right\rangle$ given in Eq. (12). These two levels display a frequency vacuum Casimir-Rabi splitting $2 \Omega_{0,3}^{2,0}$, which, as also shown in Fig. $5(\alpha)$, corresponds to the frequency of the observed Rabi-like oscillations. The small difference between the peak in Fig. 5( $\alpha)$ and $\omega_{6,5} \equiv\left(E_{6}-E_{5}\right) / \hbar=2 \Omega_{0,3}^{2,0}$ is due to the presence of the nearby higher peak at $\omega=0$. For the amplitude $A=\pi / 3$, the peak phonon number, reached just after the kick, is significantly below one, and thus the occupation probability for the state $|3\rangle_{m}$ is very low. This explains the weakness of the photonic signal in Fig. 5(a) and the smallness of the oscillations superimposed on the exponential decay in the mechanical signal $\left\langle\hat{B}^{\dagger} \hat{B}\right\rangle$. Indeed, the mechanical states $|1\rangle_{m}$ and $|2\rangle_{m}$ in the initial coherent superposition have a much larger probability than $|3\rangle_{m}$ and evolve unaffected by the vacuum field. Higher-energy mechanical states $|k\rangle_{m}$ with $k>3$ can also produce photon pairs at a rate $\Omega_{0, k}^{2, k-3}$, but their occupation probability is negligible at such a low pulse amplitude. The nonmonotonic dynamics of the signals indicates that the DCE effect is, at least partially, a reversible process: the emitted photon pairs can be reabsorbed by the moving mirror and then reemitted, if the effective DCE rates are larger than the losses. However, if one of the photons in the pair is lost, 

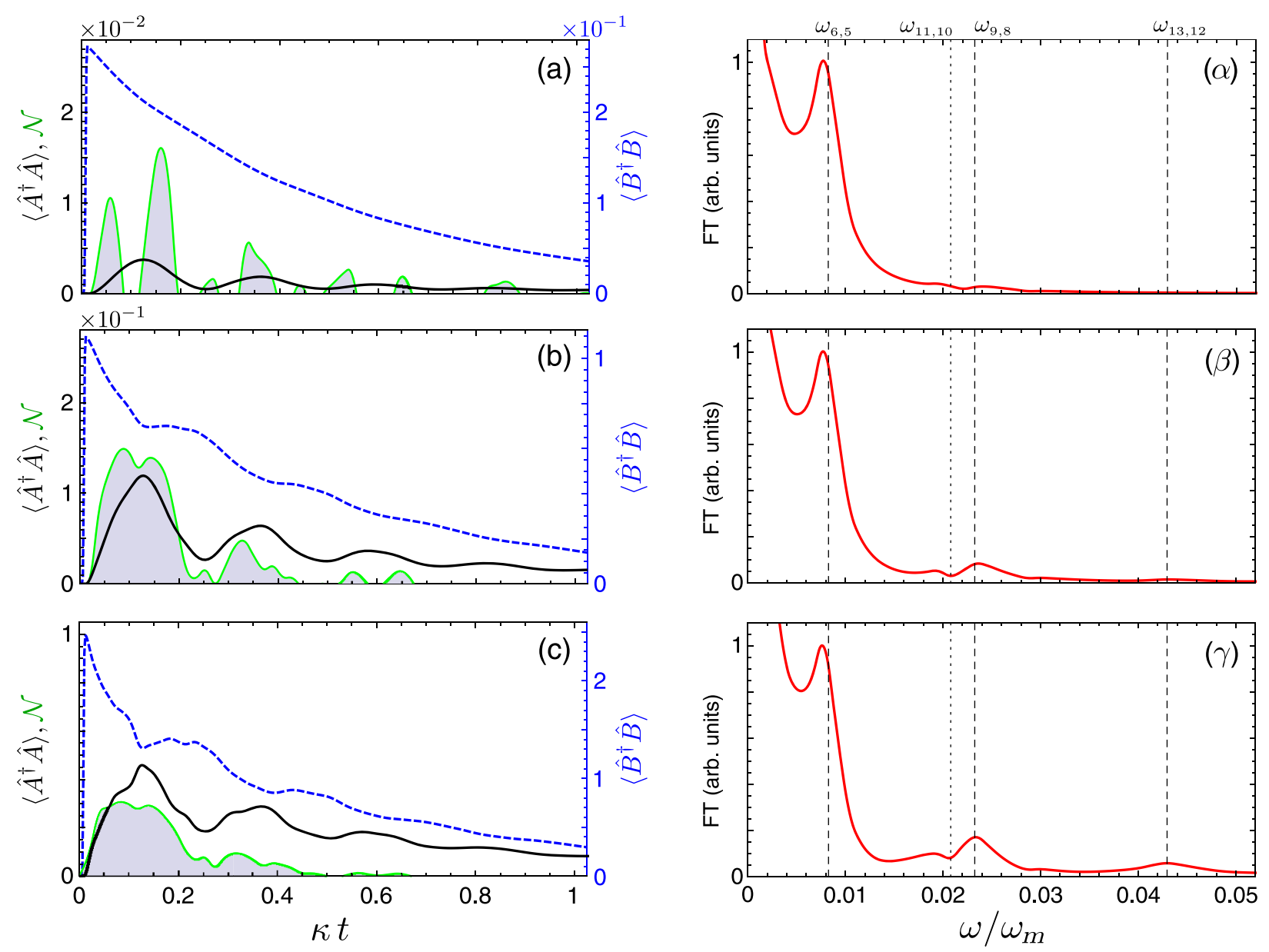

FIG. 5. System dynamics after the pulse arrival, obtained for pulses with amplitudes increasing from top to bottom: $\mathcal{A}=\pi / 3$ (a), $2 \pi / 3$ (b), and $\pi$ (c). Specifically, panels (a)-(c) display the mean intracavity photon number $\left\langle\hat{A}^{\dagger} \hat{A}\right\rangle$ (black solid curves), the mean phonon number $\left\langle\hat{B}^{\dagger} \hat{B}\right\rangle$ (blue dashed curves), and the negativity $\mathcal{N}$ (green filled curve). Panels $(\alpha)-(\gamma)$ display the Fourier transform (FT) of the mean photon number shown in the corresponding panel on the left.

the surviving one-photon state is no longer resonant with the vibrating mirror and undergoes a standard exponential decay. This effect gives rise to a decay of the oscillation amplitude faster than the signal decay.

When increasing the pulse amplitude [Figs. 5(b) and 5(c)], the mean photon number grows significantly and no longer oscillates sinusoidally. In addition, the mechanical signal deviates significantly from the exponential decay, owing to increased population of the mechanical states with phonon number $k \geq 3$ that are able to produce photon pairs.

Figures $5(\alpha)-5(\gamma)$ show the Fourier transforms of the photonic nutation signals. For $A=\pi / 3$, besides the intense peak at $\omega=0$ (describing the exponential decay induced by losses, always superimposed on the nutations), only an additional peak at $\omega \simeq \Omega_{0,3}^{2,0}$ is visible, in full agreement with the sinusoidal signal in Fig. 5(a). Increasing the pulse amplitude, a second peak at higher frequency [Fig. 5( $\beta)$ ], followed by a third at still higher frequency [Fig. 5( $\gamma$ ] appears. These two additional peaks in the Fourier transform clarify the origin of the nonsinusoidal behavior of signals in Figs. 5(b) and 5(c). They correspond to the higher-energy processes associated with the effective coupling strengths $\Omega_{0,4}^{2,1}$ and $\Omega_{0,5}^{2,2}$, both larger than $\Omega_{0,3}^{2,0}$. However, these two peak frequencies are slightly larger than the corresponding minimum half-splittings $\Omega_{0,4}^{2,1}$ and $\Omega_{0,5}^{2,2}$. This difference occurs because the ladder of vacuum Casimir-Rabi splittings, occurring at a given cavity frequency when $2 \omega_{c} \simeq 3 \omega_{m}$, is not perfectly vertical (see Fig. 2), owing to energy shifts induced by $\hat{V}_{\text {DCE }}$. Hence, if $\omega_{c}$, as in this case, is tuned to ensure that the minimum level splitting $E_{6}-E_{5}=2 \hbar \Omega_{0,3}^{2,0}$, the higher-energy split levels will not be at their minimum. The peaks clearly visible in Fig. 5( $\gamma)$ occur at frequencies $\omega=0, \omega \simeq \omega_{6,5}=2 \Omega_{0,3}^{2,0}$, $\omega \simeq \omega_{9,8}>2 \Omega_{0,4}^{2,1}$, and $\omega \simeq \omega_{13,12}>2 \Omega_{0,5}^{2,2}$. A further structure with a dip is also visible in Figs $5(\beta)$ and $5(\gamma)$ around $\omega=\omega_{11,10}$. This corresponds to the coherent coupling of the states $\left|1,3_{1}\right\rangle$ and $|3,0\rangle$, producing the eigenstates $\left|\psi_{10}\right\rangle$ and $\left|\psi_{11}\right\rangle$. These states are neither directly excited by the external mechanical pulse which generates zero-photon 
states, nor by $\hat{V}_{\text {DCE }}$, which creates or destroys photon pairs. However, the cavity losses can give rise to the decay $\left|2,3_{1}\right\rangle \rightarrow\left|1,3_{1}\right\rangle$. Hence, also the states $\left|\psi_{10}\right\rangle$ and $\left|\psi_{11}\right\rangle$ can be indirectly involved in the signal dynamics.

Analogous quantum Rabi oscillations, giving rise to discrete Fourier components, have been experimentally observed for circular Rydberg atoms in a high- $Q$ cavity [79]. In this system, however, the different level anticrossings are not affected by different energy shifts.

In cavity QED, the strong-coupling dynamics produces atom-field entanglement [80]. We investigate if this nonperturbative regime of the DCE is able to produce entanglement between the mobile mirror and the cavity field, when the mirror is excited by a coherent pulse and in the presence of mechanical and optical dissipations. The time evolution of the negativity is displayed in Figs. 5(a)-5(c). As expected, $\mathcal{N}$ increases noticeably when the pulse amplitude increases, so that the mirror dynamics is significantly affected by the DCE. We observe that, while decaying as a consequence of losses, the negativity displays a nonmonotonic behavior analogous to that observed in cavity QED [81].

\section{E. Radiative decay of a mechanical excited state}

Spontaneous emission is the process in which a quantum emitter, such as a natural or an artificial atom, or a molecule, decays from an excited state to a lower-energy state and emits a photon. This cannot be described within the classical electromagnetic theory and is fundamentally a quantum process. Here we present numerical calculations showing that a vibrating mirror prepared in an excited state (mechanical Fock state) can spontaneously emit photons like a quantum emitter. In this case, however, instead of a single photon, a photon pair is emitted. Here, instead of considering the coherent excitation of the vibrating mirror as in usual descriptions of the DCE, we assume that it is initially prepared in a Fock state. We consider the case $\omega_{c} \simeq \omega_{m}$ and the system is initialized in the state $|0,2\rangle$, with $\omega_{c}$ sufficiently detuned from the DCE resonance (minimum avoided-level crossing) at $\omega_{c}^{0} \simeq \omega_{m}$, with $\delta \omega_{c} \equiv \omega_{c}-\omega_{c}^{0}=$ $0.1 \omega_{c}^{0}$, such that the effective resonant DCE coupling is negligible. This $k=2$ mechanical Fock state can be prepared, for example, if the vibrating mirror is strongly coupled to an additional qubit [26], using the same protocols realized in circuit QED [82]. After preparation, the cavity can be quickly tuned into resonance: $\omega_{c} \rightarrow \omega_{c}^{0}$. If the cavity resonator is an $L C$ superconducting circuit, its resonance frequency can be tuned by using a SQUID. In order to not affect the mechanical Fock state during this nonadiabatic process, the tuning time must be shorter than $2 \pi / \Omega_{0,2}^{2,0}$.

Figure 6 displays the mean phonon number $\left\langle\hat{B}^{\dagger} \hat{B}\right\rangle$ (dashed blue curve), the mean intracvity photon number $\left\langle\hat{A}^{\dagger} \hat{A}\right\rangle$ (black solid curve), and the negativity (green filled
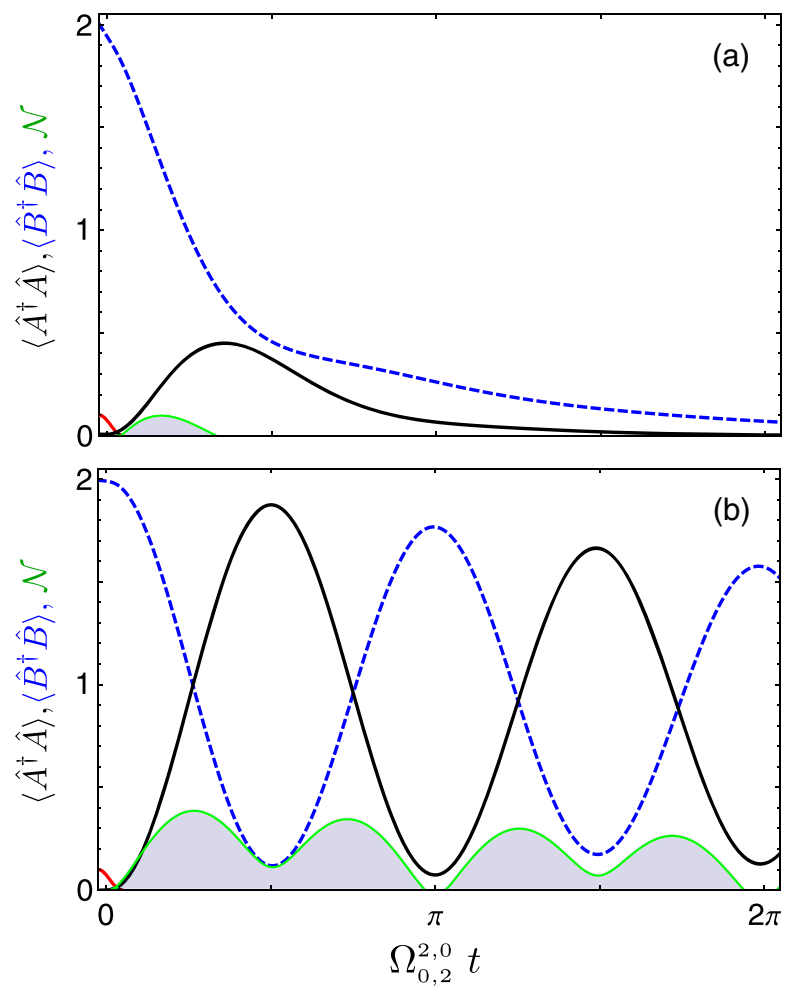

FIG. 6. Dynamics starting from a mechanical Fock state. The blue dashed curves describe the mechanical signal $\left\langle\hat{B}^{\dagger} \hat{B}\right\rangle$, while the black solid curves describe the optical signal $\left\langle\hat{A}^{\dagger} \hat{A}\right\rangle$. The green curves correspond to the negativity $\mathcal{N}$. The cavity frequency is initially detuned from the DCE resonance $\left(\delta \omega_{c}=0.1\right)$, and the system is initially prepared in the state $|0,2\rangle$. Then the cavity is quickly tuned to the DCE resonance $\left(\delta \omega_{c} \rightarrow 0\right)$. The initial detuning $\delta \omega_{c}$ is displayed as a small red solid curve in the lower left-hand corner of both panels, near $t=0$. In (a), the dynamics is evaluated in the weak-coupling regime. Panel (b) displays the vacuum Casimir-Rabi oscillations that arise when the system loss rates are low. The parameters used are provided in the text.

curve) calculated for $g / \omega_{m}=0.1$. Figure 6 also displays the initial detuning $\delta \omega_{c}$ (red solid curve). Figure 6(a), obtained using $\gamma=\Omega_{0,2}^{2,0} / 5$ and $\kappa=2.5 \gamma$, describes the irreversible mechanical decay due to both nonradiative (induced by the mechanical loss rate $\gamma$ ) and radiative decay (induced by $\hat{V}_{\text {DCE }}$ ). The radiative decay gives rise to non-negligible light emission (black solid curve), and to transient mirrorfield entanglement. Figure 6(b), obtained using the lower loss rates $\gamma=\kappa=\Omega_{0,2}^{2,0} / 80$, shows vacuum Casimir-Rabi oscillations. In this case, a photon pair can be produced at $t=\pi /\left(2 \Omega_{0,2}^{2,0}\right)$ with probability close to one.

\section{F. Analog nonperturbative DCE in all-optical systems}

The nonperturbative description of the DCE in optomechanical systems, presented here, can also be applied to other all-optical quantum systems such as parametric 
amplifiers $[8,83]$. Specifically, we consider systems operating as spontaneous parametric down-converters. A degenerate parametric amplifier (DPA) can be described by the interaction Hamiltonian (see, e.g, Ref. [84]),

$$
\hat{V}_{\mathrm{DPA}}=\lambda \hat{\Phi}_{p} \hat{\Phi}_{s}^{2}
$$

where the pump $(p)$ and signal $(s)$ field coordinates can be expressed in terms of bosonic annihilation and creation operators, $\hat{\Phi}_{p}=\Phi_{p}^{\mathrm{ZPF}}\left(\hat{b}+\hat{b}^{\dagger}\right), \Phi_{s}=\Phi_{s}^{\mathrm{ZPF}}\left(\hat{a}+\hat{a}^{\dagger}\right)$, and where $\Phi_{j}^{\mathrm{ZPF}}(j=s, p)$ is the zero-point-fluctuation amplitude of the field $\hat{\Phi}_{j}$. In most cases, when the system is used as a parametric amplifier or as a spontaneous down-converter, the pump mode is quite strongly driven at frequency $\omega_{p}$, and thus it can be considered as a classical pump field of amplitude $p$, leading to the quadratic Hamiltonian

$$
\hat{V}_{\mathrm{DPA}}=\hbar \lambda \Phi_{p}^{\mathrm{ZPF}}\left(p e^{-i \omega_{p} t}+p^{*} e^{i \omega_{p} t}\right) \hat{\Phi}_{s}^{2} .
$$

This Hamiltonian (usually further simplified discarding rapidly oscillating terms) describes many parametric processes (see, e.g., Ref. [83]), including the resonant spontaneous parametric down-conversion when $\omega_{p}=2 \omega_{s}$. However, if the pump field is weak or if the pump mode is prepared in a nonclassical state, and/or the coupling strength is sufficiently strong, this approximation fails, and the full quantum Hamiltonian Eq. (15) has to be considered. Using the bosonic operators, the resulting interaction Hamiltonian reads

$$
\hat{V}_{\mathrm{DPA}}=\frac{\hbar g}{2}\left(\hat{b}+\hat{b}^{\dagger}\right)\left(\hat{a}+\hat{a}^{\dagger}\right)^{2},
$$

which is formally identical to the optomechanical interaction in Eq. (2) $\left[g=2 \lambda \Phi_{p}^{\mathrm{ZPF}}\left(\Phi_{s}^{\mathrm{ZPF}}\right)^{2}\right]$.

This result shows that the nonperturbative analysis we present in this paper is not confined to optomechanical systems, but can also be applied to parametric amplifiers in the full quantum regime, when the pump field is not replaced by a classical amplitude and the rotating-wave approximation is not applied. According to this analysis, emission processes such as $|n, 0\rangle \rightarrow|n-q, 2\rangle$ are possible even for $q \geq 2$, if $q \omega_{p} \simeq 2 \omega_{s}$. Hence, spontaneous vacuum emission is possible even for pump frequencies lower than the signal frequency. This is a result that cannot be obtained within the usual approximate quadratic Hamiltonian in Eq. (16).

Since experiments on these all-optical systems can be simpler than experiments in optomechanical systems, they could be used as analog quantum simulators of the nonperturbative MDCE. Just as for the MDCE, significant deviations from the ordinary DCE analysis occur when the coupling strength is not too small compared to the resonance frequencies of the system. This can be difficult to achieve with parametric amplifiers at optical frequencies. However, this regime can be achieved at microwave frequencies, using superconducting quantum circuits. For example, a fully quantum device, described by the Hamiltonian in Eq. (17), could be obtained modifying flux-driven amplifiers [6,85], so that the SQUID is coupled to an additional resonator (determining the pump mode) instead of being directly driven by an external flux.

Analogous nonperturbative parametric scattering processes, requiring further theoretical analysis, can be obtained by considering nondegenerate parametric amplifiers (NDPAs) described by the interaction Hamiltonian,

$$
\hat{V}_{\mathrm{NDPA}}=\lambda \hat{\Phi}_{p} \hat{\Phi}_{s} \hat{\Phi}_{i},
$$

where $\hat{\Phi}_{i}$ is the quantum field describing the additional mode (idler). Such a nondegenerate amplifier at microwave frequencies was first proposed and realized in $2010[84,86]$.

\section{CONCLUSIONS}

We have analyzed the DCE in cavity optomechanical systems, describing quantum-mechanically both the cavity field and the vibrating mirror, fully including multiple scattering between the two subsystems. The full quantum approach developed here describes the DCE without introducing a time-dependent light-matter interaction. The only time-dependent Hamiltonian term considered in this work was the one describing the external drive of the moving mirror. Actually, we can conclude that the DCE can be described even without considering any timedependent Hamiltonian. Vacuum emission can originate from the free evolution of an initial pure mechanical excited state, in analogy with the spontaneous emission from excited atoms.

Using numerical diagonalization of the optomechanical Hamiltonian [67], including those terms usually neglected for describing current optomechanics experiments [51], we have shown that the resonant generation of photons from the vacuum is determined by a ladder of mirror-field vacuum Rabi-like splittings. These avoided-level crossings describe the energy-conserving conversion of phonons (quanta of mechanical vibration) into photon pairs. More generally, the DCE Hamiltonian in Eq. (5) describes many resonant optomechanical scattering processes $\left|n, k_{n}\right\rangle \leftrightarrow$ $\left|n+2,(k-q)_{n+2}\right\rangle$ which occur when the energies of the final and initial states coincide $\left(2 \omega_{c} \sim q \omega_{m}\right)$.

The standard resonance condition for the DCE requires a mechanical resonance frequency at least double that of the lowest mode frequency of the cavity. We have shown instead that, when the coupling between the moving mirror and the cavity field is non-negligible compared to the mechanical and optical resonance frequencies, a resonant production of photons out from the vacuum can be observed for mechanical frequencies equal to or lower than the cavity-mode frequencies. Hence, the present analysis demonstrates that optomechanical systems with coupling strengths which experiments already started to 
approach, and with vibrating mirrors working in the $\mathrm{GHz}$ spectral range, can be used to observe light emission from mechanical motion. We point out that this result originates from the unique interplay of the DCE interaction term in Eq. (5) and the standard optomechanical interaction Hamiltonian in Eq. (4). Moreover, we observe that processes satisfying $2 \omega_{c} \sim q \omega_{m}$, with $q>1$, cannot be described without including the counterrotating terms in the interaction Hamiltonian. Taking these terms into account requires a careful definition of the physical photon and phonon operators and a modified description of the dissipative dynamics, described in Appendix B.

We have also analyzed the nonperturbative regime of the DCE, which, we showed, provides direct access to the level structure determining the DCE and can display Rabi-like nutations of the cavity-field and oscillating-mirror signals. Finally, we have shown that the oscillating mirror can evolve into a state which is entangled with the radiation emitted by the mirror itself.

All the results presented here have been obtained considering a single-mode optical resonator. In most circuit-optomechanics experiments, the electromagnetic resonator is provided by a superconducting $L C$ circuit, which supports only a single mode. Considering multimode optical resonators, we observe that, for the weak-excitation regime considered here, excitation of higher-frequency modes is negligible. At higher excitation amplitudes and coupling strengths, higher-frequency modes will determine light emission at additional frequencies and should be taken into account for a quantitative analysis. In Appendix D, we provide some examples of calculations for multiple modes.

\section{ACKNOWLEDGMENTS}

F. N. was partially supported by the MURI Center for Dynamic Magneto-Optics via the AFOSR Grant No. FA9550-14-1-0040, the Japan Society for the Promotion of Science (KAKENHI), the IMPACT program of JST, JSPS-RFBR Grant No. 17-52-50023, CREST Grant No. JPMJCR1676, RIKEN-AIST Challenge Research Fund, and the Sir John Templeton Foundation. A. F. K. acknowledges support from a JSPS Postdoctoral Fellowship for Overseas Researchers (P15750).

\section{APPENDIX A: DCE MATRIX ELEMENTS}

The matrix elements $\hbar \Omega_{0, k}^{2, k-q}=\left\langle 0, k\left|\hat{V}_{\mathrm{DCE}}\right| 2,(k-q)_{2}\right\rangle$ play a key role in the MDCE, since they determine the rate at which a mechanical Fock state $|k\rangle_{m}$ can generate a photon pair. Figure 7 displays $2 \hbar \Omega_{0, k}^{2, k-q}=2\left\langle 0, k\left|\hat{V}_{\text {DCE }}\right| 2,(k-q)_{2}\right\rangle$ evaluated for $q=2,3,4$, as a function of the initial Fock state $k$, obtained for $g / \omega_{m}=0.1$ (upper panels) and 0.01 (lower panels). The two panels on the left, obtained for $q=2$, correspond to the approximate resonance condition $\omega_{c} \simeq \omega_{m}$. The center panels correspond to $\omega_{c} \simeq 1.5 \omega_{m}$, and the panels on the left correspond to $\omega_{c} \simeq 2 \omega_{m}$. Going from left to right, the matrix elements decrease. However, as long as they are comparable to the mechanical and photonic decay rates, a mechanical-optical energy exchange (at least partial) can occur, giving rise to the DCE.

The analytically calculated matrix elements Eq. (11) displayed in Fig. 7 describe the phonon-photon DCE coherent couplings obtained using first-order perturbation
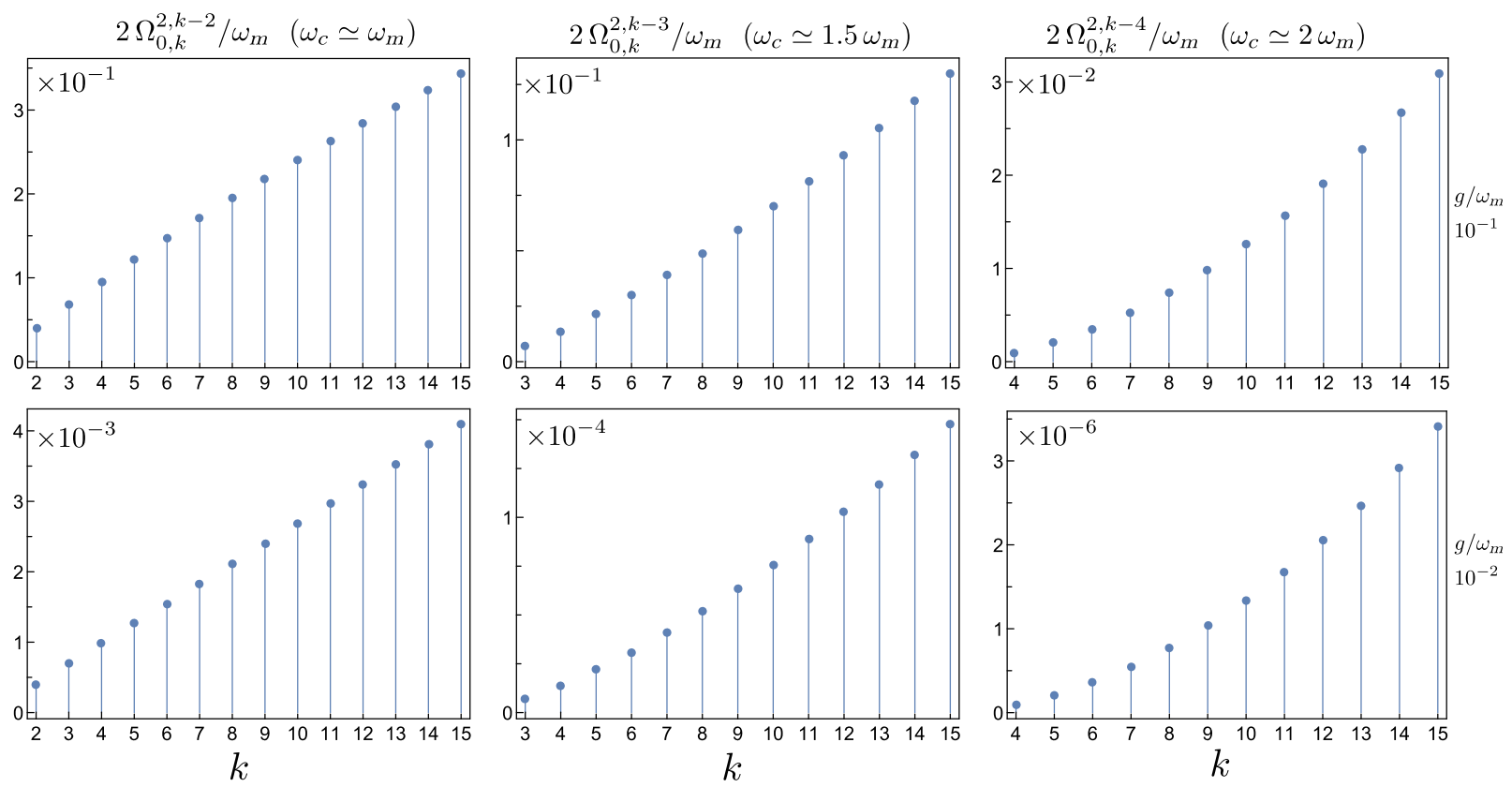

FIG. 7. Normalized effective vacuum Rabi splittings $2 \Omega_{0, k}^{2, k-q} / \omega_{m}$ of $\hat{V}_{\text {DCE }}$ between the eigenstates of $\hat{H}_{0}+\hat{V}_{\text {om }}$, evaluated for $q=2$, 3,4 , as a function of $k$. 

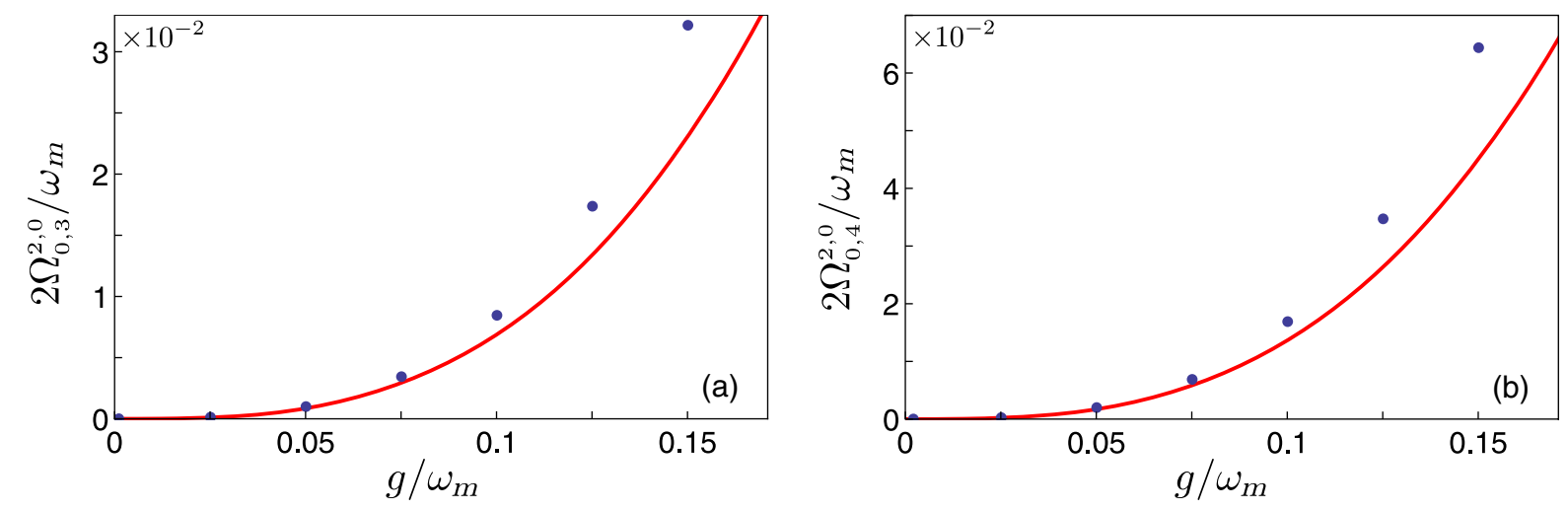

FIG. 8. Comparison between the numerically calculated normalized vacuum Rabi splitting (points) and the corresponding analytical calculations (red solid curve), obtained using first-order perturbation theory for (a) $2 \Omega_{0,3}^{2,0}$ and (b) $2 \Omega_{0,4}^{2,0}$.

theory. In order to test their accuracy, we compare them to the corresponding vacuum Casimir-Rabi splittings obtained by numerical diagonalization of $\hat{H}_{s}$ in Eq. (3). Specifically, Fig. 8 shows a comparison for $2 \Omega_{0,3}^{2,0}$ [Fig. 8(a)] and $2 \Omega_{0,4}^{2,0}$ [Fig. 8(b)], as a function of the normalized optomechanical coupling $g / \omega_{m}$. The agreement is very good for $g / \omega_{m}$ below 0.1 .

\section{APPENDIX B: MASTER EQUATION}

We take into account dissipation and decoherence effects by adopting a master-equation approach. For strongly coupled hybrid quantum systems, the description offered by the standard quantum-optical master equation breaks down $[53,87]$. Following Refs. $[52,53,72]$, we express the system-bath interaction Hamiltonian in the basis formed by the energy eigenstates of $\hat{H}_{s}$. By applying the standard Markov approximation and tracing out the reservoir d.o.f., we arrive at the master equation for the density-matrix operator $\hat{\rho}(t)$ :

$\hat{\rho}(t)=\frac{i}{\hbar}\left[\hat{\rho}(t), \hat{H}_{s}+\hat{V}_{m}(t)\right]+\kappa \mathcal{D}[\hat{A}] \hat{\rho}(t)+\gamma \mathcal{D}[\hat{B}] \hat{\rho}(t)$.

Here, the constants $\kappa$ and $\gamma$ correspond to the cavity-field and mirror damping rates. The dressed photon and phonon lowering operators $\hat{O}=\hat{A}, \hat{B}$ are defined in terms of their bare counterparts $\hat{o}=\hat{a}, \hat{b}$ as [88]

$$
\hat{O}=\sum_{E_{n}>E_{m}}\left\langle\psi_{m}\left|\left(\hat{o}+\hat{o}^{\dagger}\right)\right| \psi_{n}\right\rangle\left|\psi_{m}\right\rangle\left\langle\psi_{n}\right|,
$$

where $\left|\psi_{n}\right\rangle(n=0,1,2, \ldots)$ are the eigenvectors of $\hat{H}_{s}$ and $E_{n}$ the corresponding eigenvalues. The superoperator $\mathcal{D}$ is defined as

$$
\mathcal{D}[\hat{O}] \hat{\rho}=\frac{1}{2}\left(2 \hat{O} \hat{\rho} \hat{O}^{\dagger}-\hat{O}^{\dagger} \hat{O} \hat{\rho}-\hat{\rho} \hat{O}^{\dagger} \hat{O}\right) .
$$

The spectrum and the eigenstates of $\hat{H}_{s}$ are obtained by standard numerical diagonalization in a truncated finite-dimensional Hilbert space. The truncation is realized by including only the lowest-energy $N_{c}$ photonic and $N_{m}$ mechanical Fock states. These truncation numbers are chosen in order to ensure that the lowest $M<N_{c} \times N_{m}$ energy eigenvalues and the corresponding eigenvectors, which are involved in the dynamical processes investigated here, are not significantly affected when increasing $N_{c}$ and $N_{m}$. Then the density matrix in the basis of the system eigenstates is truncated in order to exclude all the higherenergy eigenstates which are not populated during the dynamical evolution. This truncation, of course, depends on the excitation strength $\mathcal{F}(t)$ in Eq. (6). The system of differential equations resulting from the master equation is then solved by using a standard Runge-Kutta method with step control. In this way, the mechanical-optical quantum correlations are taken into account to all significant orders.

In writing the master equation, we assume that the baths are at zero temperature. The generalization to $T \neq 0$ reservoirs can be derived following Ref. [53]. Note that the photonic and mechanical lowering operators involve only transitions from higher- to lower-energy states. If $\hat{V}_{\text {DCE }}$ is neglected, $\hat{A}=\hat{a}$ and $\hat{B}=\hat{b}-\left(g / \omega_{m}\right) \hat{a}^{\dagger} \hat{a}$. Following Ref. [72], the master equation [Eq. (B1)] has been derived without making the post-trace rotating-wave approximation used in Ref. [87], which is not applicable in the presence of equally spaced (even approximately) energy levels, as in the present case.

\section{APPENDIX C: NEGATIVITY}

Negativity is an entanglement monotone and does not increase under local operations and classical communication [89]. Hence, it represents a proper measure of entanglement, although it can be zero even if the state is entangled, for a specific class of entangled states [89]. The negativity of a subsystem $A$ can be defined as the absolute sum of the negative eigenvalues of the partial transpose $\rho^{T_{A}}$ of the density matrix $\rho$ with respect to a subsystem $A$ : $\mathcal{N}(\rho)=\sum_{i}\left(\left|\lambda_{i}\right|-\lambda_{i}\right) / 2$, where $\lambda_{i}$ are the eigenvalues of 
$\rho^{T_{A}}$. In this case, the subsystems $A$ and $B$ are the cavity field and the vibrating mirror, respectively.

\section{APPENDIX D: NUMERICAL RESULTS INCLUDING AN ADDITIONAL ELECTROMAGNETIC MODE}

In order to understand the influence of higher-energy modes on the DCE quantum dynamics, here we present numerical calculations obtained including a second cavity mode at higher frequency. Specifically, we consider the case where the second mode frequency is twice that of the first mode: $\omega_{2}=2 \omega_{1}$. This situation occurs for a $\lambda / 2$ optical resonator $\left(\omega_{1}=\pi v / l\right.$, where $v$ is the speed of light and $l$ the resonator length). The total Hamiltonian is

$$
\hat{H}_{s}=\hat{H}_{0}+\hat{H}_{I}
$$

where

$$
\hat{H}_{0}=\sum_{j=1,2} \hbar \omega_{j} \hat{a}_{j}^{\dagger} \hat{a}_{j}+\hbar \omega_{m} \hat{b}^{\dagger} \hat{b}
$$

and

$$
\hat{H}_{I}=\frac{\hbar g}{2}\left[\sum_{j=1,2} \sqrt{\frac{\omega_{j}}{\omega_{1}}}(-1)^{j}\left(\hat{a}_{j}+\hat{a}_{j}^{\dagger}\right)\right]^{2}\left(\hat{b}+\hat{b}^{\dagger}\right)
$$

We use $g / \omega_{m}=5 \times 10^{-2}$ and explore the region $\omega_{1} \simeq \omega_{m}$. We also use a driving amplitude $A / \gamma=1$ and decay rate for the two modes $\kappa_{1}=\kappa_{2}=\kappa=3 \times 10^{-3} \omega_{m}$, and $\gamma=12 \kappa$.

Figure 9 (left-hand panel) displays the lowest-energy levels of the system Hamiltonian as a function of the ratio between the cavity frequency $\omega_{1}$ (note that $\omega_{2}=2 \omega_{1}$ ) and the mechanical frequency $\omega_{m}$ in the spectral region around $\omega_{1} \simeq \omega_{m}$. The lowest-energy anticrossing (violet circle) appears (as in the case with a single mode described in the main text) around $\omega / \omega_{m}=2$ (on the vertical axis). It originates from the hybridization of the state $|0,0,2\rangle$ (here, the first entry describes the photon number of mode 1 , the second describes the photon number of mode 2 , and the third, the phonon number) corresponding to the flat horizontal level (outside the anticrossing region) with the state $|2,0,0\rangle$. The minimum splitting occurs at
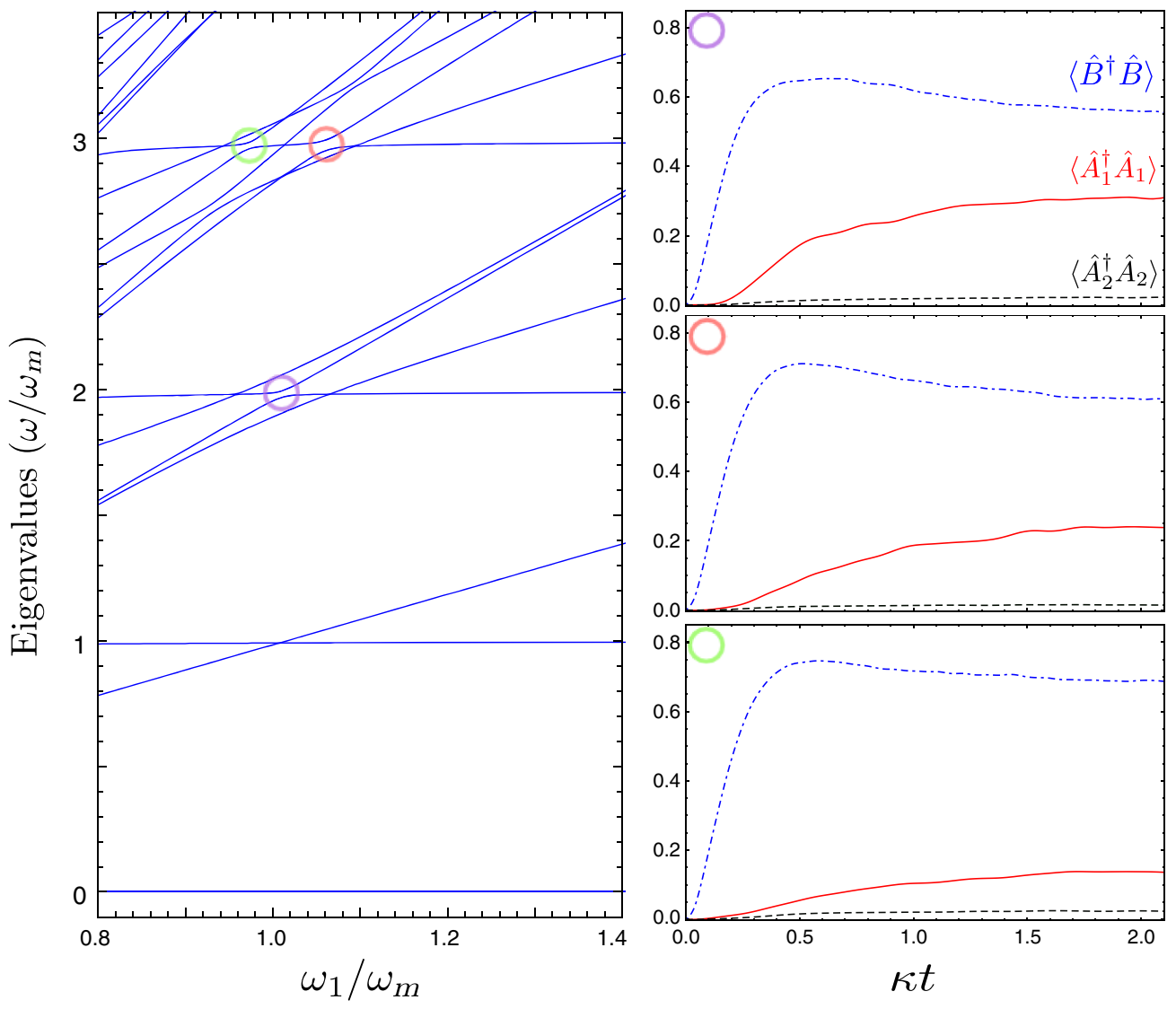

FIG. 9. Left: Lowest-energy levels of the system Hamiltonian as a function of the ratio between the bare resonance frequency of mode $1 \omega_{1}$ and the mechanical frequency $\omega_{m}$. Right: System dynamics for $\omega_{1} \simeq \omega_{m}$ under continuous-wave drive of the vibrating mirror. The blue dash-dotted curves describe the mean phonon number $\left\langle\hat{B}^{\dagger} \hat{B}\right\rangle$, while the red solid curves describe the mean intracavity photon number of mode $1\left\langle\hat{A}_{1}^{\dagger} \hat{A}_{1}\right\rangle$, and the dashed black curves describe the mean intracavity photon number of mode $2\left\langle\hat{A}_{2}^{\dagger} \hat{A}_{2}\right\rangle$. The top panel has been obtained using $\omega_{1} / \omega_{m} \simeq 1.01$, the center panel using $\omega_{1} / \omega_{m} \simeq 1.06$, and the bottom panel using $\omega_{1} / \omega_{m} \simeq 0.972$. 
$\omega_{1} / \omega_{m} \simeq 1.01$. The other two levels present in the region that do not display avoided-level crossing effects correspond to the states $|1,0,1\rangle$ and $|0,1,0\rangle$.

The second mode gives rise to hybridization effects only at higher energies, starting from $\omega / \omega_{m}=3$. At this energy, the state $|0,0,3\rangle$ hybridizes with the state $|2,0,1\rangle$ (indicated by the red circle). The minimum splitting occurs at $\omega_{1} / \omega_{m} \simeq 1.06$. Also in this case, mode 2 does not contribute. However, the state $|0,0,3\rangle$ also hybridizes with the state $|1,1,0\rangle$ (green circle). In this case, the annihilation of three phonons can give rise to the production of a photon pair with one photon in mode 1 and the other in mode 2. The minimum splitting, in this case, is obtained at $\omega_{1} / \omega_{m} \simeq 0.972$. We observe that the avoided-level crossing minimum for this last case (involving the second mode) occurs at a cavity frequency that is different from those of the other two avoided-level crossings involving mode 1 only. Hence, tuning the cavity frequency to $\omega_{1} / \omega_{m} \simeq 1.01$ (or $\omega_{1} / \omega_{m} \simeq 1.06$ ), we expect almost negligible contributions from mode 2. At higher energy (not shown here) the state $|0,0,4\rangle$ hybridizes with the state $|0,2,0\rangle$. However, at low driving amplitudes, the probability to excite four phonons can be very low.

In order to directly study the influence of the second mode on the DCE, we carried out simulations of the dynamics. The results have been obtained by including 50 energy eigenstates. The panels on the right-hand side in Fig. 9 display the time evolution of the mean values of the phonon and photon numbers in the different modes. The system starts from its ground state, and the mechanical oscillator is coherently driven at $\omega_{d}=\omega_{m}$ with drive amplitude $A / \gamma=1$. The upper panel corresponds to $\omega_{1} / \omega_{m} \simeq 1.01$. We observe that, as expected, the population of mode 2 is very small compared to that of mode 1 . The middle panel on the right-hand side has been obtained using $\omega_{1} / \omega_{m} \simeq 1.06$. Also in this case, the population of mode 2 is very small compared to that of mode 1 . In the lower panel (obtained using $\omega_{1} / \omega_{m} \simeq 0.972$ ), the mean photon number for mode 2 increases a little bit, although it remains small, while that of mode 1 decreases.

In conclusion, in all three cases the mean photon number of mode 2 is much smaller than that of mode 1 . Moreover, outside the resonance region where mode 2 photons can be produced $\left(\omega_{1} / \omega \simeq 0.972\right)$, the production of mode 2 photons is negligible. In any case, even increasing the excitation amplitude, the main effect of including other modes is to obtain new resonance conditions which may determine the emission of photons at different frequencies.

[1] J. Schwinger, On Gauge Invariance and Vacuum Polarization, Phys. Rev. 82, 664 (1951).

[2] G. T. Moore, Quantum Theory of the Electromagnetic Field in a Variable-Length One-Dimensional Vavity, J. Math. Phys. (N.Y.) 11, 2679 (1970).
[3] S. W. Hawking, Black Hole Explosions, Nature (London) 248, 30 (1974).

[4] J. Schwinger, Casimir Light: The Source, Proc. Natl. Acad. Sci. U.S.A. 90, 2105 (1993).

[5] E. Yablonovitch, Accelerating Reference Frame for Electromagnetic Waves in a Rapidly Growing Plasma: UnruhDavies-Fulling-DeWitt Radiation and the Nonadiabatic Casimir Effect, Phys. Rev. Lett. 62, 1742 (1989).

[6] C. M. Wilson, G. Johansson, A. Pourkabirian, M. Simoen, J. R. Johansson, T. Duty, F. Nori, and P. Delsing, Observation of the Dynamical Casimir Effect in a Superconducting Circuit, Nature (London) 479, 376 (2011).

[7] P. Lähteenmäki, G. S. Paraoanu, J. Hassel, and P. J. Hakonen, Dynamical Casimir Effect in a Josephson Metamaterial, Proc. Natl. Acad. Sci. U.S.A. 110, 4234 (2013).

[8] P. D. Nation, J. R. Johansson, M. P. Blencowe, and Franco Nori, Colloquium: Stimulating Uncertainty: Amplifying the Quantum Vacuum with Superconducting Circuits, Rev. Mod. Phys. 84, 1 (2012).

[9] S. W. Hawking, Particle Creation by Black Holes, Commun. Math. Phys. 43, 199 (1975).

[10] W. G. Unruh, Notes on Black-Hole Evaporation, Phys. Rev. D 14, 870 (1976).

[11] S. A. Fulling and P. C. W. Davies, Radiation from a Moving Mirror in Two Dimensional Space-Time: Conformal Anomaly, Proc. R. Soc. A 348, 393 (1976).

[12] V. V. Dodonov, Current Status of the Dynamical Casimir Effect, Phys. Scr. 82, 038105 (2010).

[13] E. Sassaroli, Y. N. Srivastava, and A. Widom, Photon Production by the Dynamical Casimir Effect, Phys. Rev. A 50, 1027 (1994).

[14] A. Lambrecht, M.-T. Jaekel, and S. Reynaud, Motion Induced Radiation from a Vibrating Cavity, Phys. Rev. Lett. 77, 615 (1996).

[15] V. V. Dodonov and A. B. Klimov, Generation and Detection of Photons in a Cavity with a Resonantly Oscillating Boundary, Phys. Rev. A 53, 2664 (1996).

[16] G. Schaller, R. Schützhold, G. Plunien, and G. Soff, Dynamical Casimir Effect in a Leaky Cavity at Finite Temperature, Phys. Rev. A 66, 023812 (2002).

[17] W.-J. Kim, J. H. Brownell, and R. Onofrio, Detectability of Dissipative Motion in Quantum Vacuum via Superradiance, Phys. Rev. Lett. 96, 200402 (2006).

[18] Yu. E. Lozovik, V. G. Tsvetus, and E. A. Vinogradov, Femtosecond Parametric Excitation of Electromagnetic Field in a Cavity, JETP Lett. 61, 723 (1995).

[19] M. Uhlmann, G. Plunien, R. Schützhold, and G. Soff, Resonant Cavity Photon Creation via the Dynamical Casimir Effect, Phys. Rev. Lett. 93, 193601 (2004).

[20] M. Crocce, D. A. R. Dalvit, F. C. Lombardo, and F. D. Mazzitelli, Model for Resonant Photon Creation in a Cavity with Time-Dependent Conductivity, Phys. Rev. A 70, 033811 (2004).

[21] C. Braggio, G. Bressi, G. Carugno, C. Del Noce, G. Galeazzi, A. Lombardi, A. Palmieri, G. Ruoso, and D. Zanello, A Novel Experimental Approach for the Detection of the Dynamical Casimir Effect, Europhys. Lett. 70, 754 (2005).

[22] E. Segev, B. Abdo, O. Shtempluck, E. Buks, and B. Yurke, Prospects of Employing Superconducting Stripline 
Resonators for Studying the Dynamical Casimir Effect Experimentally, Phys. Lett. A 370, 202 (2007).

[23] S. De Liberato, C. Ciuti, and I. Carusotto, Quantum Vacuum Radiation Spectra from a Semiconductor Microcavity with a Time-Modulated Vacuum Rabi Frequency, Phys. Rev. Lett. 98, 103602 (2007).

[24] J. R. Johansson, G. Johansson, C. M. Wilson, and F. Nori, Dynamical Casimir Effect in a Superconducting Coplanar Waveguide, Phys. Rev. Lett. 103, 147003 (2009).

[25] J. R. Johansson, G. Johansson, C. M. Wilson, and F. Nori, Dynamical Casimir Effect in Superconducting Microwave Circuits, Phys. Rev. A 82, 052509 (2010).

[26] A. D. O'Connell, M. Hofheinz, M. Ansmann, R. C. Bialczak, M. Lenander, E. Lucero, M. Neeley, D. Sank, H. Wang, M. Weides et al., Quantum Ground State and Single-Phonon Control of a Mechanical Resonator, Nature (London) 464, 697 (2010).

[27] S. De Liberato, D. Gerace, I. Carusotto, and C. Ciuti, Extracavity Quantum Vacuum Radiation from a Single Qubit, Phys. Rev. A 80, 053810 (2009).

[28] L. Garziano, A. Ridolfo, R. Stassi, O. Di Stefano, and S. Savasta, Switching On and Off of Ultrastrong Light-Matter Interaction: Photon Statistics of Quantum Vacuum Radiation, Phys. Rev. A 88, 063829 (2013).

[29] L. Garziano, R. Stassi, A. Ridolfo, O. Di Stefano, and S. Savasta, Vacuum-Induced Symmetry Breaking in a Superconducting Quantum Circuit, Phys. Rev. A 90, 043817 (2014).

[30] D. Hagenmüller, All-Optical Dynamical Casimir Effect in a Three-Dimensional Terahertz Photonic Band Gap, Phys. Rev. B 93, 235309 (2016).

[31] O. Di Stefano, R. Stassi, L. Garziano, A. F. Kockum, S. Savasta, and F. Nori, Feynman-Diagrams Approach to the Quantum Rabi Model for Ultrastrong Cavity QED: Stimulated Emission and Reabsorption of Virtual Particles Dressing a Physical Excitation, New J. Phys. 19, 053010 (2017).

[32] M. Cirio, K. Debnath, N. Lambert, and F. Nori, Amplified Optomechanical Transduction of Virtual Radiation Pressure, Phys. Rev. Lett. 119, 053601 (2017).

[33] S. De Liberato, Virtual Photons in the Ground State of a Dissipative System, Nat. Commun. 8, 1465 (2017).

[34] J. Q. You and F. Nori, Superconducting Circuits and Quantum Information, Phys. Today 58, No. 11, 42 (2005).

[35] J. Q. You and F. Nori, Atomic Physics and Quantum Optics Using Superconducting Circuits, Nature (London) 474, 589 (2011).

[36] Z.-L. Xiang, S. Ashhab, J. Q. You, and F. Nori, Hybrid Quantum Circuits: Superconducting Circuits Interacting with Other Quantum Systems, Rev. Mod. Phys. 85, 623 (2013).

[37] X. Gu, A. F. Kockum, A. Miranowicz, Y-x. Liu, and F. Nori, Microwave Photonics with Superconducting Quantum Circuits, Phys. Rep. 718-719, 1 (2017).

[38] W. H. Louisell, A. Yariv, and A. E. Siegman, Quantum Fluctuations and Noise in Parametric Processes. I., Phys. Rev. 124, 1646 (1961).

[39] S. E. Harris, M. K. Oshman, and R. L. Byer, Observation of Tunable Optical Parametric Fluorescence, Phys. Rev. Lett. 18, 732 (1967).
[40] F. X. Dezael and A. Lambrecht, Analogue Casimir Radiation Using an Optical Parametric Oscillator, Europhys. Lett. 89, 14001 (2010).

[41] P. D. Noerdlinger, Boundary Conditions for Moving Magnetic Fields and Lorentz. Transformation of Surface Currents, Am. J. Phys. 39, 191 (1971).

[42] M. Kira, W. Hoyer, and S. W. Koch, Terahertz Signatures of the Exciton Formation Dynamics in Non-Resonantly Excited Semiconductors, Solid State Commun. 129, 733 (2004).

[43] G. Plunien, R. Schützhold, and G. Soff, Dynamical Casimir Effect at Finite Temperature, Phys. Rev. Lett. 84, 1882 (2000).

[44] J. Haro and E. Elizalde, Hamiltonian Approach to the Dynamical Casimir Effect, Phys. Rev. Lett. 97, 130401 (2006).

[45] V. V. Dodonov, Dynamical Casimir Effect in a Nondegenerate Cavity with Losses and Detuning, Phys. Rev. A 58, 4147 (1998).

[46] M.-T. Jaekel and S. Reynaud, Quantum Fluctuations of Position of a Mirror in Vacuum, J. Phys. I (France) 3, 1 (1993).

[47] P. A. Maia Neto and S. Reynaud, Dissipative Force on a Sphere Moving in Vacuum, Phys. Rev. A 47, 1639 (1993).

[48] M. Kardar and R. Golestanian, The Friction, of Vacuum, and Other Fluctuation-Induced Forces, Rev. Mod. Phys. 71, 1233 (1999).

[49] M. F. Maghrebi, R. Golestanian, and M. Kardar, Scattering Approach to the Dynamical Casimir Effect, Phys. Rev. D 87, 025016 (2013).

[50] D. A. R. Dalvit and P. A. Maia Neto, Decoherence via the Dynamical Casimir Effect, Phys. Rev. Lett. 84, 798 (2000).

[51] M. Aspelmeyer, T. J. Kippenberg, and F. Marquardt, Cavity Optomechanics, Rev. Mod. Phys. 86, 1391 (2014).

[52] H.-P. Breuer and F. Petruccione, The Theory of Open Quantum Systems (Oxford University Press, New York, 2002).

[53] D. Hu, S.-Y. Huang, J.-Q. Liao, L. Tian, and H.-S. Goan, Quantum Coherence in Ultrastrong Optomechanics, Phys. Rev. A 91, 013812 (2015).

[54] J. D. Teufel, D. Li, M. S. Allman, K. Cicak, A. J. Sirois, J. D. Whittaker, and R.W. Simmonds, Circuit Cavity Electromechanics in the Strong-Coupling Regime, Nature (London) 471, 204 (2011).

[55] J. Chan, T. P. M. Alegre, A. H. Safavi-Naeini, J. T. Hill, A. Krause, S. Gröblacher, M. Aspelmeyer, and O. Painter, Laser Cooling of a Nanomechanical Oscillator into Its Quantum Ground State, Nature (London) 478, 89 (2011).

[56] A. J. Rimberg, M. P. Blencowe, A. D. Armour, and P. D. Nation, A Cavity-Cooper Pair Transistor Scheme for Investigating Quantum Optomechanics in the Ultra-Strong Coupling Regime, New J. Phys. 16, 055008 (2014).

[57] T. T. Heikkilä, F. Massel, J. Tuorila, R. Khan, and M. A. Sillanpää, Enhancing Optomechanical Coupling via the Josephson Effect, Phys. Rev. Lett. 112, 203603 (2014).

[58] J.-M. Pirkkalainen, S. U. Cho, F. Massel, J. Tuorila, T. T. Heikkilä, P. J. Hakonen, and M. A. Sillanpää, Cavity Optomechanics Mediated by a Quantum Two-Level System, Nat. Commun. 6, 6981 (2015). 
[59] P. D. Nation, J. Suh, and M. P. Blencowe, Ultrastrong Optomechanics Incorporating the Dynamical Casimir Effect, Phys. Rev. A 93, 022510 (2016).

[60] F. Rouxinol, Y. Hao, F. Brito, A. O. Caldeira, E. K. Irish, and M. D. LaHaye, Measurements of Nanoresonator-Qubit Interactions in a Hybrid Quantum Electromechanical System, Nanotechnology 27, 364003 (2016).

[61] J.-Q. Liao, K. Jacobs, F. Nori, and R. W. Simmonds, Modulated Electromechanics: Large Enhancements of Nonlinearities, New J. Phys. 16, 072001 (2014).

[62] A. Nunnenkamp, K. Børkje, and S. M. Girvin, SinglePhoton Optomechanics, Phys. Rev. Lett. 107, 063602 (2011).

[63] L. Garziano, R. Stassi, V. Macrí, S. Savasta, and O. Di Stefano, Single-Step Arbitrary Control of Mechanical Quantum States in Ultrastrong Optomechanics, Phys. Rev. A 91, 023809 (2015).

[64] K. Stannigel, P. Komar, S. J. M. Habraken, S. D. Bennett, M. D. Lukin, P. Zoller, and P. Rabl, Optomechanical Quantum Information Processing with Photons and Phonons, Phys. Rev. Lett. 109, 013603 (2012).

[65] V. Macrí, L. Garziano, A. Ridolfo, O. Di Stefano, and S. Savasta, Deterministic Synthesis of Mechanical NOON States in Ultrastrong Optomechanics, Phys. Rev. A 94, 013817 (2016).

[66] B. E. Ordaz-Mendoza and S. F. Yelin, A Novel Regime for the Dynamical Casimir Effect, arXiv:1612.02525.

[67] C. K. Law, Interaction between a Moving Mirror and Radiation Pressure: A Hamiltonian Formulation, Phys. Rev. A 51, 2537 (1995).

[68] S. Butera and R. Passante, Field Fluctuations in a OneDimensional Cavity with a Mobile Wall, Phys. Rev. Lett. 111, 060403 (2013).

[69] F. Armata, M. S. Kim, S. Butera, L. Rizzuto, and R. Passante, Nonequilibrium Dressing in a Cavity with a Movable Reflecting Mirror, Phys. Rev. D 96, 045007 (2017).

[70] S. Haroche, Nobel Lecture: Controlling Photons in a Box and Exploring the Quantum to Classical Boundary, Rev. Mod. Phys. 85, 1083 (2013).

[71] T. Niemczyk, F. Deppe, H. Huebl, E. P. Menzel, F. Hocke, M. J. Schwarz, J. J. Garcia-Ripoll, D. Zueco, T. Hümmer, E. Solano, A. Marx, and R. Gross, Circuit Quantum Electrodynamics in the Ultrastrong-Coupling Regime, Nat. Phys. 6, 772 (2010).

[72] K. K. W. Ma and C. K. Law, Three-Photon Resonance and Adiabatic Passage in the Large-Detuning Rabi Model, Phys. Rev. A 92, 023842 (2015).

[73] L. Garziano, R. Stassi, V. Macrì, A. F. Kockum, S. Savasta, and F. Nori, Multiphoton Quantum Rabi Oscillations in Ultrastrong Cavity QED, Phys. Rev. A 92, 063830 (2015).

[74] L. Garziano, V. Macrì, R. Stassi, O. Di Stefano, F. Nori, and S. Savasta, One Photon Can Simultaneously Excite Two or More Atoms, Phys. Rev. Lett. 117, 043601 (2016).
[75] A. F. Kockum, A. Miranowicz, V. Macrì, S. Savasta, and F. Nori, Deterministic Quantum Nonlinear Optics with Single Atoms and Virtual Photons, Phys. Rev. A 95, 063849 (2017).

[76] R. Stassi, V. Macrì, A. F. Kockum, O. Di Stefano, A. Miranowicz, S. Savasta, and F. Nori, Quantum Nonlinear Optics without Photons, Phys. Rev. A 96, 023818 (2017).

[77] A. Megrant, C. Neill, R. Barends, B. Chiaro, Y. Chen, L. Feigl, J. Kelly, E. Lucero, M. Mariantoni, P. J. J. O’Malley et al., Planar Superconducting Resonators with Internal Quality Factors Above One Million, Appl. Phys. Lett. 100, 113510 (2012).

[78] J. R. Johansson, G. Johansson, C. M. Wilson, P. Delsing, and F. Nori, Nonclassical Microwave Radiation from the Dynamical Casimir Effect, Phys. Rev. A 87, 043804 (2013).

[79] M. Brune, F. Schmidt-Kaler, A. Maali, J. Dreyer, E. Hagley, J. M. Raimond, and S. Haroche, Quantum Rabi Oscillation: A Direct Test of Field Quantization in a Cavity, Phys. Rev. Lett. 76, 1800 (1996).

[80] J. M. Raimond, M. Brune, and S. Haroche, Manipulating Quantum Entanglement with Atoms and Photons in a Cavity, Rev. Mod. Phys. 73, 565 (2001).

[81] B. Bellomo, R. Lo Franco, and G. Compagno, NonMarkovian Effects on the Dynamics of Entanglement, Phys. Rev. Lett. 99, 160502 (2007).

[82] M. Hofheinz, H. Wang, M. Ansmann, R. C. Bialczak, E. Lucero, M. Neeley, A. D. O’Connell, D. Sank, J. Wenner, J. M. Martinis, and A. N. Cleland, Synthesizing Arbitrary Quantum States in a Superconducting Resonator, Nature (London) 459, 546 (2009).

[83] L. Mandel and E. Wolf, Optical Coherence and Quantum Optics (Cambridge University Press, Cambridge, England, 1995).

[84] N. Bergeal, R. Vijay, V. E. Manucharyan, I. Siddiqi, R. J. Schoelkopf, S. M. Girvin, and M. H. Devoret, Analog Information Processing at the Quantum Limit with a Josephson Ring Modulator, Nat. Phys. 6, 296 (2010).

[85] T. Yamamoto, K. Inomata, M. Watanabe, K. Matsuba, T. Miyazaki, W. D. Oliver, Y. Nakamura, and J. S. Tsai, FluxDriven Josephson Parametric Amplifier, Appl. Phys. Lett. 93, 042510 (2008).

[86] N. Bergeal, F. Schackert, M. Metcalfe, R. Vijay, V.E. Manucharyan, L. Frunzio, D. E. Prober, R. J. Schoelkopf, S. M. Girvin, and M.H. Devoret, Phase Preserving Amplification Near the Quantum Limit with a Josephson Ring Modulator, Nature (London) 465, 64 (2010).

[87] F. Beaudoin, J. M. Gambetta, and A. Blais, Dissipation and Ultrastrong Coupling in Circuit QED, Phys. Rev. A 84, 043832 (2011).

[88] A. Ridolfo, M. Leib, S. Savasta, and M. J. Hartmann, Photon Blockade in the Ultrastrong Coupling Regime, Phys. Rev. Lett. 109, 193602 (2012).

[89] G. Vidal and R.F. Werner, Computable Measure of Entanglement, Phys. Rev. A 65, 032314 (2002). 